\title{
A metric Kan-Thurston theorem
}

\author{
Ian J. Leary
}

\begin{abstract}
For every simplicial complex $X$, we construct a locally CAT(0) cubical complex $T_{X}$, a cellular isometric involution $\tau$ on $T_{X}$ and a map $t_{X}: T_{X} \rightarrow X$ with the following properties: $t_{X} \tau=t_{X}$; $t_{X}$ is a homology isomorphism; the induced map from the quotient space $T_{X} /\langle\tau\rangle$ to $X$ is a homotopy equivalence; the induced map from the fixed point space $T_{X}^{\tau}$ to $X$ is a homology isomorphism. The construction is functorial in $X$.

One corollary is an equivariant Kan-Thurston theorem: every connected proper $G$-CWcomplex has the same equivariant homology as the classifying space for proper actions, E $\widetilde{G}$, of some other group $\widetilde{G}$. From this we obtain extensions of a theorem of Quillen on the spectrum of a (Borel) equivariant cohomology ring and a result of Block concerning assembly conjectures. Another corollary of our main result is that there can be no algorithm to decide whether a $\mathrm{CAT}(0)$ cubical group is generated by torsion.

In appendices we prove some foundational results concerning cubical complexes, including the infinite dimensional case. We characterize the cubical complexes for which the natural metric is complete; we establish Gromov's criterion for infinite-dimensional cubical complexes; we show that every $\mathrm{CAT}(0)$ cube complex is cubical; we deduce that the second cubical subdivision of any locally $\mathrm{CAT}(0)$ cube complex is cubical.
\end{abstract}

\section{Introduction}

In [16, D. Kan and W. Thurston showed that every simplicial complex $X$ has the same homology as some aspherical space $T_{X}$, or equivalently every connected simplicial complex $X$ has the same homology as the classifying space for some discrete group $G_{X}$. More precisely, they give a map $t_{X}: T_{X} \rightarrow X$ which induces a homology isomorphism for any local coefficient system on $X$. Furthermore, the construction of $T_{X}$ and $t_{X}$ has good naturality properties. The Kan-Thurston theorem has been extended by a number of authors $\mathbf{3}, \mathbf{1 3}, \mathbf{2 2}$. In particular, Baumslag Dyer and Heller showed that in the case when $X$ is finite, the space $T_{X}$ may be taken to be a finite simplicial complex [3. (In contrast, the original $T_{X}$ was uncountable whenever $X$ had dimension at least two.)

The Kan-Thurston theorem has also been generalized in a number of ways. McDuff showed that every $X$ has the homotopy type of the classifying space of some discrete monoid $M_{X}[\mathbf{2 3}$. Leary and Nucinkis showed that every $X$ has the homotopy type of a space of the form $\underline{E} G / G$ for some discrete group $G$, where $\underline{\mathrm{E}} G$ denotes the classifying space for proper actions of $G[\mathbf{1 9}]$.

Our purpose is to give new proofs of some of these results using methods that come from metric geometry. We give an extremely short summary of these methods before stating our result. A good general reference for this material is $[\mathbf{8}$.

A geodesic metric space is a metric space in which any two points are joined by a 'geodesic', i.e., a path of length equal to their distance. A geodesic metric space is CAT(0) if any geodesic triangle is at least as thin as a comparison triangle in the Euclidean plane having the same side lengths. Any $\mathrm{CAT}(0)$ space $X$ is contractible: for any point $x \in X$ one may define a contraction

2000 Mathematics Subject Classification 20F67 (primary), 20J06, 55P99, 57S30 (secondary).

Partially supported by NSF grant DMS-0804226 and by the Heilbronn Institute. Some of this work was done at MSRI, Berkeley, where research is supported by NSF grant DMS-0441170. 
of $X$ to $x$ by moving points at constant speed along the (unique) geodesic path joining them to $x$.

Let $T$ be a cubical complex. The link of any vertex in $T$ is naturally a simplicial complex, and vertex links in the universal cover $\widetilde{T}$ are isomorphic to vertex links in $T$. There is a metric on $T$ in which distances are defined in terms of the lengths of piecewise-linear paths, using an isomorphism between each cube of $T$ and a standard Euclidean cube of side length one to measure the length of paths. Gromov has given a simple criterion for this metric to be CAT(0): this happens if and only if $T$ is simply connected and the link of each vertex is a flag complex [8, 11. In particular, it follows that if all vertex links in $T$ are flag complexes, then $T$ is aspherical.

The standard references for Gromov's criterion consider only the case when $T$ is finitedimensional (see [8, theorem II.5.20], [11, section 4.2.C], [7, ch. 2] or the unpublished [25] for example). In Theorem B.8 in an appendix to this paper we give a proof in the general case.

Let $T$ be a locally $\operatorname{CAT}(0)$ cube complex. There are many known constraints on the fundamental group $G$ of such a $T$. For example, if $T$ is finite, then $G$ is bi-automatic, which implies that there are good algorithms to solve the word and conjugacy problems in $G[\mathbf{2 7}$. As another example, when $T$ is countable, the group $G$ has the Haagerup property, i.e., $G$ admits a proper affine isometric action on a Hilbert space. This implies that $G$ can contain no infinite subgroup having Kazhdan's property (T) [26].

In contrast, we show that there is no restriction whatsoever on the homology of a locally $\mathrm{CAT}(0)$ cubical complex. We also show that there is no restriction on the homotopy type of the quotient of a locally CAT(0) cubical complex by an isometric cellular involution, apart from the obvious condition that it should be homotopy equivalent to a $\mathrm{CW}$-complex. The following statement is a version of our main theorem.

Theorem A. Let $X$ be a simplicial complex. There is a locally CAT(0) cubical complex $T_{X}$ and a map $t_{X}: T_{X} \rightarrow X$ with the following properties.

(i) The map $t_{X}$ induces an isomorphism on homology for any local coefficients on $X$.

(ii) There is an isometric cellular involution $\tau$ on $T_{X}$ so that $t_{X} \circ \tau=t_{X}$ and the induced map $T_{X} /\langle\tau\rangle \rightarrow X$ is a homotopy equivalence.

(iii) The map $t_{X}: T_{X}^{\tau} \rightarrow X$ induces an isomorphism on homology for any local coefficients on $X$, where $T_{X}^{\tau}$ denotes the fixed point set in $T_{X}$ for the action of $\tau$.

If $X$ is finite, then so is $T_{X}$. The construction is functorial in the following senses:

- Any simplicial map $f: X \rightarrow Y$ that is injective on each simplex gives rise to a $\tau$-equivariant cubical map $T_{f}: T_{X} \rightarrow T_{Y}$.

- In the case when $f$ is injective, $T_{f}$ embeds $T_{X}$ isometrically as a totally geodesic subcomplex of $T_{Y}$.

- In the case when $f$ is locally injective, $T_{f}$ is a locally isometric map. In particular, in this case $T_{f}$ is injective on fundamental groups.

If $X$ is the union of subcomplexes $X_{\alpha}$, then $T_{X}$ is equal to the union of the $T_{X_{\alpha}}$. The dimension of $T_{X}$ is equal to the dimension of $X$, except that when $X$ is 2-dimensional, $T_{X}$ is 3 -dimensional. In all cases, the dimension of $T_{X}^{\tau}$ is equal to the dimension of $X$.

By a locally injective map of simplicial complexes, we mean a map $f: X \rightarrow Y$ which is injective on each simplex of $X$, and for each vertex $x \in X$ induces an injective map from the link of $x$ to the link of $f(x)$. Equivalently, a locally injective map of simplicial complexes is a simplicial map which induces a locally injective map of realizations.

The existence of $T_{X}$ and $t_{X}$ having property (i) is a strengthening of the Baumslag-DyerHeller version of the Kan-Thurston theorem [3, 16], since the only groups used are fundamental groups of locally CAT(0) cubical complexes. Similarly, the existence of $T_{X}$ and $t_{X}$ having property (ii) is a strengthening of the theorem of Leary and Nucinkis on possible homotopy 
types of $\mathrm{E} G / G$ since the only groups used are groups that act with stabilizers of order one and two on $\operatorname{CAT}(0)$ cubical complexes $[\mathbf{1 9}$. This follows from the fact that whenever a group $G$ acts with finite stabilizers on a CAT(0) cubical complex $X$ that cubical complex is a model for $\underline{E} G$ (see Theorem B.11).

We describe a number of corollaries to Theorem A in Sections 811 of this paper. We believe that none of these results follow from earlier versions of the Kan-Thurston theorem, and we hope that they go some way towards motivating our work.

One advantage of Theorem A over other versions of the Kan-Thurston theorem is that it readily yields an equivariant version, which we state as Theorem 8.3. If $G$ acts on $X$ which we suppose to be connected, then $G$ acts also on $T_{X}$. Let $E$ be the universal covering space of $T_{X}$, and let $\widetilde{G}$ be the group of self-homeomorphisms of $E$ that lift the action of $G$ on $T_{X}$. If we assume that $G$ acts on $X$ with finite stabilizers, then $\widetilde{G}$ acts with finite stabilizers on the $\operatorname{CAT}(0)$ cubical complex $E$. It follows (see Theorem B.11) that $E$ is a model for $\underline{E} \widetilde{G}$, the classifying space for proper actions of $\widetilde{G}$. By construction, there is a surjective group homomorphism $\widetilde{G} \rightarrow G$, and an equivariant map $\underline{\mathrm{E}} \widetilde{G} \cong E \rightarrow X$ which is an equivariant homology isomorphism.

Our construction of $t_{X}$ and $T_{X}$ having property 1 is closely modelled on the construction of groups used by Maunder in $2 \mathbf{2 2}$ and by Baumslag, Dyer and Heller in $\mathbf{3}$. The main ingredients in the proof in 3 can be illustrated by solving a simpler problem: suppose that one wants to construct, for each $n>0$, a group $G_{n}$ so that the integral homology of $G_{n}$ is isomorphic to the homology of the $n$-sphere. To solve this problem, they fix an acyclic group $H$, together with an element $h \in H$ of infinite order. Take $G_{1}$ to be the subgroup of $H$ generated by $h$, and define groups $H_{n}$ (to play the role of the $n$-disc) and $G_{n}$ for $n \geq 2$ as follows. Let $H_{2}=H$, our fixed acyclic group. Now define $G_{2}$ by gluing two 2-discs along a circle: $G_{2}=H *_{G_{1}} H$. Define $H_{3}=H_{2} *_{G_{1}}\left(G_{1} \times H\right)$, and check that $H_{3}$ is an acyclic group containing $G_{2}$ as a subgroup. (The standard copy of $H_{2}$ and its conjugate by $(1, h) \in G_{1} \times H$ together generate a subgroup of $H_{3}$ isomorphic to $G_{2}$.) The definitions of the higher groups are now clear: $G_{n+1}=H_{n+1} *_{G_{n}} H_{n+1}$ and $H_{n+1}=H_{n} *_{G_{n-1}}\left(G_{n-1} \times H\right)$.

This construction carries over without change to the metric world, once one has found an acyclic locally $\mathrm{CAT}(0)$ cubical complex $A$ to replace the classifying space for $H$. The proof from 22 when translated into the metric world gives a construction of the space denoted by $T_{X}^{\tau}$ in the statement of Theorem A. To obtain $T_{X}$ and the involution $\tau$, we need a second acyclic locally $\mathrm{CAT}(0)$ cubical complex $A^{\prime}$, containing $A$ and equipped with an involution $\tau$ such that $A$ is equal to the fixed point set $A^{\prime \tau}$ and such that the quotient space $A^{\prime} /\langle\tau\rangle$ is contractible. Roughly speaking, $T_{X}$ is constructed by attaching a copy of $A^{\prime}$ to every copy of $A$ that appears in the construction of $T_{X}^{\tau}$. The acyclic locally CAT(0) 2-complex $A$ that we construct is a presentation 2-complex, in which the 2-cells are large, right-angled polygons which in turn are made from smaller squares.

The remainder of this paper is structured as follows. In Section 2 we consider the problem of making a CAT(0) polygon with given side lengths from unit squares. In Section 3 we build acyclic locally CAT(0) 2-complexes that will be used in the construction of $A$ and $A^{\prime}$. Section 4 constructs $A, A^{\prime}$ and $\tau$ as described in the previous paragraph, and Section 5 gives the proof of Theorem A, following [22].

Sections [ and 7 consider variants on the construction of $T_{X}$, including a cube complex whose cubical subdivision is $T_{X}$, a version $T_{X}^{\prime}$ which is always metrically complete but for which the map $T_{X}^{\prime} \rightarrow X$ is not necessarily proper, and a version $\widehat{T}_{X}$ defined for arbitrary spaces $X$, and such that the map from $\widehat{T}_{X} /\langle\tau\rangle$ to $X$ is a weak homotopy equivalence. Section 8 contains Theorem 8.3. an equivariant Kan-Thurston theorem for actions with finite stabilizers. Sections 9 and 10 discuss applications of Theorem 8.3 to Borel equivariant cohomology and to assembly conjectures. In particular, Corollary 9.2 is an extension to Quillen's theorem on the spectrum of a Borel equivariant cohomology ring [29, and Theorem 10.1 generalizes an observation of 
Block [5, Introduction]. Block's observation concerned the Baum-Connes conjecture for torsionfree groups, while our Theorem 10.1 applies to groups with torsion and to many assembly conjectures. In Section 11 we use Theorem A to deduce that there can be no algorithm to decide whether a $\operatorname{CAT}(0)$ cubical group is generated by torsion. Section 12 discusses some open questions related to our work.

Sections $\mathrm{A}$, and Contain results about cubical complexes with an emphasis on the infinitedimensional case, and should be viewed as appendices to the paper. In some cases the results for finite-dimensional cubical complexes are well-known, and our proofs proceed by reducing the arbitrary case to the finite-dimensional case. In Theorem A.6 we characterize the cubical (and simplicial) complexes for which the natural path metric is complete. In Theorem B.8 we establish Gromov's criterion: a simply-connected cubical complex is CAT(0) if and only if all links are flag complexes. In Theorem B.11 we show that whenever a finite group acts on a CAT(0) cubical complex (not assumed to be metrically complete), the fixed point set is nonempty. Section $\mathrm{C}$ concerns the comparison between cubical complexes and the more general complexes often called cube complexes. The result is that in the $\operatorname{CAT}(0)$ case there is almost no advantage to using cube complexes rather than cubical complexes. In Theorem C.4 we show that every CAT $(0)$ cube complex is in fact cubical. In Corollary C.11 we deduce that the second cubical subdivision of every locally CAT $(0)$ cube complex is cubical.

The results in Sections $\mathrm{A}, \mathrm{B}$ and $\mathrm{C}$, will not be surprising to experts, but we know of no published proof the main results that covers the infinite-dimensional case. In a private communication in 2007, Michah Sageev told the author that Yael Algom-Kfir had obtained a proof of Gromov's criterion for infinite-dimensional cube complexes (proved here as Theorem B.8) while working on her MSc with him. Also in 2007, Yael Algom-Kfir told the author that she did not intend to publish her proof. Our proof of Theorem B.8 involves a reduction to the case of a finite-dimensional cubical complex, and we rely heavily on results of Sageev [31] in making this reduction.

Acknowledgements. This work was started in 2001, when the author visited Wolfgang Lück in Münster and Jean-Claude Hausmann in Geneva. Some progress was made at MSRI in 2007, and the author thanks these people and institutes for their hospitality. The author also thanks many other people for helpful conversations concerning this work, especially Graham Niblo, Boris Okun and Michah Sageev. Finally, the author thanks Raeyong Kim and Chris Hruska for their helpful comments on earlier version of this paper.

\section{Tesselating $C A T(0)$ polygons}

We seek CAT(0) tesselations by squares of polygons with given side lengths. A tesselated $n$-gon is defined to be a 2 -dimensional cubical complex $S$ which is homeomorphic to a disc, together with an ordered sequence of $n$ distinct vertices $v_{1}, \ldots, v_{n}$ on the boundary circle $\partial S$ of $S$. It will be convenient to view the index set for these vertices as $\mathbb{Z} / n$ rather than $\mathbb{Z}$, so that $v_{n+k}=v_{k}$. Call the vertices $v_{i}$ the corners of the polygon, and call the complementary pieces of $\partial S$ the (open) sides of the polygon. The $i$ th side is the piece whose closure contains $v_{i}$ and $v_{i+1}$. The $i$ th side length $l_{i}$ is defined to be the number of edges in the $i$ th side.

Suppose that $\left(S, v_{1}, \ldots, v_{n}\right)$ is a tesselated polygon. For $v$ a vertex of $S$, let $n(v)$ denote the degree of $v$, i.e., the number of neighbouring vertices. Define the curvature $c(v)$ of $S$ at $v$ to be

$$
c(v)= \begin{cases}4-n(v) & \text { if } v \notin \partial S \\ 3-n(v) & \text { if } v \in \partial S \text { is not a corner of } S \\ 2-n(v) & \text { if } v \text { is a corner of } S\end{cases}
$$


We say that $\left(S, v_{1}, \ldots, v_{n}\right)$ is a CAT(0) tesselated polygon if for every vertex $v \in S, c(v) \leq 0$. By Gromov's criterion, it follows that the natural path-metric on $S$ is a CAT(0) metric in which each of the sides of $S$ is a geodesic.

An easily proved combinatorial version of the Gauss-Bonnet theorem shows that for any $n$-gon $S$,

$$
\sum_{v \in S} c(v)=4-n
$$

It follows easily that the only tesselated CAT $(0)$ quadrilaterals are rectangles with side lengths $\left(l, l^{\prime}, l, l^{\prime}\right)$, since a $\operatorname{CAT}(0)$ quadrilateral $S$ must have $c(v)=0$ for all $v \in S$. Similarly, a tesselated $\operatorname{CAT}(0)$ pentagon can have $c(v) \neq 0$ for just one vertex $v$.

Clearly, some restrictions are required on the side lengths $l_{i}$ for the construction of a $\operatorname{CAT}(0)$ $n$-gon. For example, the perimeter $p$, defined by $p=\sum_{i} l_{i}$ must be even since every edge not in $\partial S$ belongs to exactly two squares. Note also it cannot be the case that any $l_{i}$ is greater than or equal to $p / 2$, since each side of the polygon is a geodesic. We do not characterize the sequences $\left(l_{1}, \ldots, l_{n}\right)$ for which $S$ exists, but just give some constructions that suffice for our purposes.

First we give three ways to obtain a new $\operatorname{CAT}(0)$ polygon, starting from a $\operatorname{CAT}(0) n$-gon with side lengths $\left(l_{1}, \ldots, l_{n}\right)$.

One can introduce a new corner in the middle of the $i$ th side of the $n$-gon, to produce an $(n+1)$-gon: if $k$ satisfies $0<k<l_{i}$ then an $(n+1)$-gon with side lengths $\left(l_{1}, \ldots, l_{i-1}, k, l_{i}-\right.$ $\left.k, \ldots, l_{n}\right)$ is obtained.

One can add a $1 \times l_{i}$ rectangle to the $i$ th side of the $n$-gon, producing a new $n$-gon with side lengths $\left(l_{1}, \ldots, l_{i-1}+1, l_{i}, l_{i+1}+1, \ldots, l_{n}\right)$. By applying this process once for each $i$, one obtains an $n$-gon with side lengths $\left(l_{1}+2, \ldots, l_{n}+2\right)$ consisting of the original $n$-gon with a 'collar' attached.

One can take the cubical subdivision of an $n$-gon, producing an $n$-gon with side lengths double those of the original $n$-gon.

Next we discuss two ways to build a $\mathrm{CAT}(0)$ polygon, starting just from the required side lengths.

Suppose that $R$ is a Euclidean rectangle tesselated by squares, with perimeter $p$ and corners $w_{1}, \ldots, w_{4}$. Any choice of $n$ vertices $v_{1}, \ldots, v_{n}$ in $\partial R$ which gives the required side lengths gives a way to view $R$ as a tesselated $n$-gon. However, this $n$-gon will fail to be $\operatorname{CAT}(0)$ if $\left\{w_{1}, \ldots, w_{4}\right\} \nsubseteq\left\{v_{1}, \ldots, v_{n}\right\}$. Define subrectangles $R_{1}^{\prime}, \ldots, R_{4}^{\prime}$ of $R$ as follows: if there exists $i$ such that $w_{j}=v_{i}$, then let $R_{j}^{\prime}$ be the single point $w_{j}$; if $w_{j}$ lies in the interior of the $i$ th side of $R$, let $R_{j}^{\prime}$ be the subrectangle of $R$ with $w_{j}, v_{i}$ and $v_{i+1}$ as three of its vertices. Provided that the four rectangles $R_{1}^{\prime}, \ldots, R_{4}^{\prime}$ are disjoint, we define $S$ to be the closure in $R$ of $R-\bigcup_{j=1}^{4} R_{j}^{\prime}$. This $S$ is a $\operatorname{CAT}(0) n$-gon with the required side lengths. Note that the curvature of $S$ is concentrated along $\partial S$, since each interior vertex of $S$ has valency 4 .

For example, consider the problem of realizing a pentagon with side lengths $(2,2,1,2,1)$. If we start with a square of side 2 , there are two distinct ways to cut up the boundary. One of these does not work, since two of the subrectangles $R_{j}^{\prime}$ and $R_{j+1}^{\prime}$ corresponding to adjacent vertices meet. The other way gives a valid $S$ in the form of an ' $L$ '-shape consisting of three squares.

As a second example, consider the problem of realizing a hexagon with sides $(2,1,1,2,1,1)$. Again, there are two ways to break up the boundary of a square $R$ of side 2 , one of which gives $S=R$, with four of the hexagon's vertices coincident with vertices of $R$ and two on the midpoints of opposite edges. The other way does not work, since for some $j$ the subrectangles $R_{j}^{\prime}$ and $R_{j+2}^{\prime}$ meet, and so the construction gives a bow-tie consisting of two squares joined at a vertex. The defect with this 'hexagon' is that the midpoints of the two long sides coincide. 


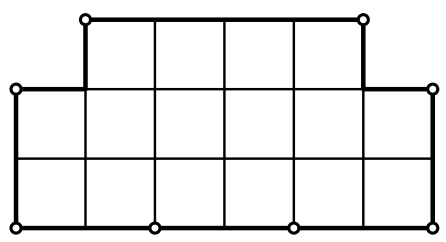

Figure 1. A CAT(0) octagon

There is however another way to realize the hexagon with sides $(2,1,1,2,1,1)$, as a suitably labelled $1 \times 3$ rectangle.

As a third example, Figure 1 illustrates a CAT(0) octagon with seven sides of length 2 and one side of length 4 that was constructed in this way. Note that the curvature in this octagon is concentrated at two of its corners and at the midpoints of two of its sides.

One corollary of this construction is that every sequence $\left(l_{1}, \ldots, l_{n}\right)$ with $p$ divisible by 4 and each $l_{i}$ strictly less than $p / 8$ can be realized: the condition on the lengths $l_{i}$ guarantees that if we choose $R$ to be a square, then the four rectangles $R_{j}^{\prime}$ are disjoint.

Next consider the problem of constructing a $\operatorname{CAT}(0) n$-gon $S$ in which all the curvature is concentrated at a single vertex. In the case when the vertex $v$ with non-zero curvature is in the interior of $S$, this is equivalent to identifying the sides of $n$ rectangles $R_{1}, \ldots, R_{n}$ where $R_{i}$ has side lengths $k_{i}$ and $k_{i+1}$. (As usual the index $i$ should be assumed to lie in the integers modulo $n$.) This gives rise to a CAT(0) $n$-gon with side lengths $l_{i}$ given by the equations $l_{i}=k_{i}+k_{i+2}$. The cases when the vertex with non-zero curvature is in $\partial S$ arise as degenerate cases. If $k_{i+1}=0$ but $k_{j}>0$ for $j \neq i$, then the vertex with non-zero curvature lies in the interior of the $i$ th side of the $n$-gon. If $k_{i}=k_{i+1}=0$ but $k_{j}>0$ for $j \neq i, i+1$, then the curvature is concentrated at the corner $v_{i}$ of $S$. Other degenerate cases do not give rise to $\operatorname{CAT}(0) n$-gons.

The equations $l_{i}=k_{i}+k_{i+2}$ can be solved uniquely for $k_{i}$ if and only if $n$ is not divisible by 4. If $n=4 m$, there is a solution if and only if

$$
\sum_{i=1}^{m} l_{4 i-3}=\sum_{i=1}^{m} l_{4 i-1} \quad \text { and } \quad \sum_{i=1}^{m} l_{4 i-2}=\sum_{i=1}^{m} l_{4 i} .
$$

If we define $m$ by $m=n$ in the case when $n$ is odd, and $m=n / 2$ when $n$ is congruent to 2 modulo 4 , then the unique solution in these cases is given by

$$
k_{i}=\frac{1}{2} \sum_{j=0}^{m-1}(-1)^{j} l_{i+2 j} .
$$

At least in the case when 4 does not divide $n$, this solves completely the question of whether there exists a CAT(0) $n$-gon with curvature at just one vertex and side lengths $\left(l_{1}, \ldots, l_{n}\right)$. The above equation computes $k_{1}, \ldots, k_{n}$, and then there is such an $n$-gon if and only if each $k_{i}$ is a positive integer, and either at most one $k_{i}$ is equal to zero or there exists $i$ such that $k_{j}=0$ if and only if $j=i$ or $j=i+1$. Since every CAT(0) pentagon has curvature at just one vertex, this completely solves the question of the possible side lengths of CAT $(0)$ pentagons.

Figure 2 shows three CAT(0) hexagons that can be built in this way by identifying the sides of six Euclidean rectangles.

\section{Acyclic 2-complexes}

We give some constructions for acyclic locally $\mathrm{CAT}(0)$ 2-complexes. In each case we start with a balanced finite group presentation. We take a rose in which each petal has the same length, and attach right-angled CAT(0) 2-cells as constructed in Section 2 Since we wish to 

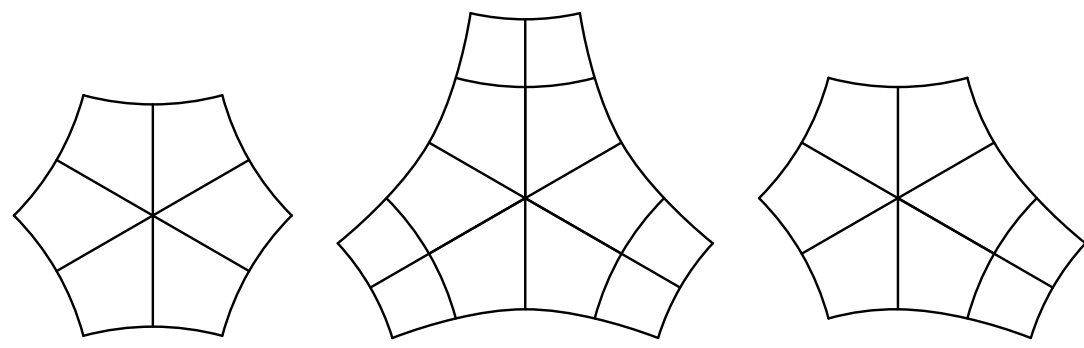

Figure 2. Three CAT(0) hexagons

build our right-angled polygons using unit squares, the lengths of the petals of the roses that we use will be larger; petals of length 4 will suffice.

Fix integers $m$ and $n$ with $3 \leq m \leq n$, and construct a 2-complex $Y$ by attaching $2 n$ polygons with $2 m$ sides to a rose $Y^{1}$ with $2 n$ petals. The petals of the rose will be labelled $a_{i}$ and $b_{i}$ for $i \in \mathbb{Z} / n$. The $2 m$ sides of the polygons will be labelled not by a single letter, but instead by a longer word. To describe the process it is convenient to let $A_{i}$ stand for a reduced word in the letters $\left\{a_{1}, \ldots, a_{n}\right\}$ and their inverses which begins and ends with $a_{i}$. For example, $a_{1} a_{2}^{-2} a_{3} a_{1}$ is a valid choice for $A_{1}$, whereas $a_{1}^{-1} a_{3}^{2} a_{1}$ is not. Similarly, let $B_{i}$ denote a reduced word in the letters $\left\{b_{1}, \ldots, b_{n}\right\}$ and their inverses which begins and ends with $b_{i}$. Define a meeting point to be a pair consisting of a word $a_{i}^{\epsilon_{1}} b_{j}^{\epsilon_{2}}$ and its formal inverse $b_{j}^{-\epsilon_{1}} a_{i}^{-\epsilon_{2}}$, where $1 \leq i, j \leq n$ and $\epsilon_{1}, \epsilon_{2} \in\{ \pm 1\}$, so that there are $4 n^{2}$ distinct meeting points. The sides of the polygons will be labelled by words $A_{i}$ and $B_{j}$ of the types described above, or their inverses, in such a way that at the meeting points appearing at the $4 m n \leq 4 n^{2}$ corners of the polygons are all distinct. It is clear that there are many ways to do this. Two such labellings will suffice for our purposes, the $2 n$ words

$$
A_{1} B_{j} A_{2} B_{j+1} A_{3} \cdots A_{m} B_{j+m-1} \quad \text { and } \quad A_{1} B_{j}^{-1} A_{2} B_{j-1}^{-1} A_{3} \cdots A_{m} B_{j-m+1}
$$

for $j \in \mathbb{Z} / n$ or the $2 n$ words

$$
A_{1} B_{j} A_{2} B_{j} \cdots A_{m} B_{j} \quad \text { and } \quad A_{1} B_{j}^{-1} A_{2} B_{j}^{-1} A_{3} \cdots A_{m} B_{j}^{-1}
$$

for $j \in \mathbb{Z} / n$. In each case, the different appearances of each $A_{i}$ and $B_{j}$ should be read as representing possibly different words of the allowed type, rather than lots of copies of the same word.

The subwords $A_{i}$ and $B_{j}$ occurring in the $2 n$ words that represent the boundaries of the polygons should be chosen in such a way that there is a tesselated CAT $(0)$ polygon whose side lengths are four times the word lengths $\left|A_{i}\right|$ and $\left|B_{j}\right|$. For example, if $m \geq 5$ and all of the words $A_{i}$ and $B_{j}$ are of approximately equal lengths, then such tesselated CAT(0) polygons can always be made by cutting the corners off a Euclidean rectangle as described in the previous section.

The 2-complex $Y$ is built by starting with a rose $Y^{1}$ with $2 n$ petals, each built from four 1-cells. Each petal is labelled by one of the letters $a_{i}$ or $b_{i}$. Now the $2 n$ tesselated $\operatorname{CAT}(0)$ polygons are attached using the $2 n$ words described above. To check that this 2-complex is locally CAT(0), we check the link of each vertex. To simplify the proof, we consider only the case when the curvature in each of the $2 n$ polygons is concentrated at interior vertices. The links of vertices in the interiors of the polygons are circles of circumference at least $2 \pi$, and so cause no problems. Similarly, the link of any vertex in $Y^{1}$, apart from the central vertex of the rose, consists of two points joined by a number of arcs each of length $\pi$. It remains to consider the central vertex of the rose. The link of this vertex is a graph with $4 n$ vertices, the inward and outward ends of the $2 n$ petals of the rose, which we shall denote by $a_{i}^{(i)}, a_{i}^{(o)}, b_{i}^{(i)}$ and $b_{i}^{(o)}$ 
for $i \in \mathbb{Z} / n$. There are $4 m n$ edges in this graph of length $\pi / 2$, coming from the corners of the $2 n$-gons, and a large number of edges of length $\pi$, coming from the interiors of the sides of the $2 n$-gons. For each $i, j \in \mathbb{Z} / n$ and each $x, y \in\{i, o\}$, there is at most one short edge between $a_{i}^{(x)}$ and $b_{j}^{(y)}$. Thus the short edges form a subgraph of the complete bipartite graph $K^{2 n, 2 n}$. Each long edge either has both of its vertices in $\left\{a_{i}^{(x)}: i \in \mathbb{Z} / n, x \in\{i, o\}\right\}$ or both of its vertices in $\left\{b_{i}^{(x)}: i \in \mathbb{Z} / n, x \in\{i, o\}\right\}$. The fact that the words along the sides of the $2 n$-gons are reduced implies that no single long edge is a loop. Hence the shortest loops in the link graph consist of either two long edges, one long and two short edges, or four short edges. It follows that the 2-complex $Y$ is locally $\operatorname{CAT}(0)$.

The 2-complex $Y$ will be acyclic if and only if the abelianization of its fundamental group is trivial. This of course places extra conditions on the words along the edges of the $2 n$-gons. We state some results that can be proved using this process.

REMARK 3.1. Note that the subcomplex of $Y$ consisting of the 0 -cell and the 1-cells labelled ' $a_{i}$ ' is a totally geodesic subcomplex, since the points $a_{i}^{(x)}$ in the vertex link in $Y$ are separated by at least $\pi$. It follows that the subgroup of $\pi_{1}(Y)$ generated by $a_{1}, \ldots, a_{n}$ is a free group on these $n$ generators. Similarly, the subgroup of $\pi_{1}(Y)$ generated by $b_{1}, \ldots, b_{n}$ is a free group freely generated by these elements.

Proposition 3.2. The group presented on generators $a, \ldots, f$ subject to the following 6 relators is non-trivial, torsion-free and acyclic. The corresponding presentation 2-complex may be realized as a non-positively curved square complex (or as a negatively curved polygonal complex).

$$
\begin{gathered}
a b c d e f, \quad a b^{-1} c^{2} f^{-1} e^{2} d^{-1}, \\
a d^{-2} c b^{-2} e f^{-1}, \quad a d^{2} c f^{2} e b^{2}, \quad a f^{-2} c d^{-1} e b^{-2} .
\end{gathered}
$$

Proof. Here $n=3$, and we have written $a, c, e$ in place of $a_{1}, a_{2}, a_{3}$ and similarly $b, d, f$ in place of $b_{1}, b_{2}, b_{3}$. Each word $A_{i}$ and $B_{j}$ consists of a power of a single letter. Using techniques from Section 2, one shows easily that $\mathrm{CAT}(0)$ hexagons with side lengths $(4,4,4,4,4,4)$, $(8,4,8,4,4,4)$ and $(8,4,8,4,8,4)$ can be built from unit squares, and these suffice to make this 2-complex: in fact, the cubical subdivisions of the three hexagons shown in Figure 2 can be used for this purpose. The claim in parentheses follows from the fact that obtuse hexagons with side lengths in these ratios can be realized in the hyperbolic plane.

Proposition 3.3. There is a non-contractible finite locally CAT $(0)$ acyclic 2-dimensional cubical complex $Y$ which admits a cubical involution $\tau: Y \rightarrow Y$ such that

(i) The fixed point set $Y^{\tau}$ consists of a single point;

(ii) The quotient space $Y / \tau$ is contractible;

(iii) $Y$ contains an isometrically embedded $\tau$-invariant 2-petalled rose with $\tau$ acting by swapping the two petals.

Proof. Let $n=4$, and for $i \in \mathbb{Z} / n$ define

$$
A_{i}=a_{i} a_{i+2} a_{i}^{-2} a_{i+2}^{-1} a_{i}, \quad B_{i}=b_{i} b_{i+2} b_{i}^{-2} b_{i+2}^{-1} b_{i} .
$$

These words $A_{i}$ and $B_{i}$ will be fixed throughout this proof. Now consider the $2 n$ words

$$
a_{i} A_{i} B_{i} A_{i+1} B_{i} A_{i+2} B_{i} A_{i+3} B_{i} \quad \text { and } \quad b_{i} B_{i} A_{i}^{-1} B_{i} A_{i+1}^{-1} B_{i} A_{i+2}^{-1} B_{i} A_{i+3}^{-1} .
$$


The techniques of Section 2 enable one to construct a CAT(0) octagon with each side length 24 except for one side of length 28. For example, one could take the CAT(0) tesselated octagon depicted in Figure 1 and collar it five times to produce a CAT(0) octagon with seven sides of length 12 and one of length 14 . The cubical subdivision of this octagon is the octagon that we require. Attach 8 copies of this octagon to an 8 -petalled rose (with petals labelled by the 8 generators) to make the 2-complex $Y$.

There is an action of $\mathbb{Z} / 4$ on the eight generators, with $1 \in \mathbb{Z} / 4$ acting by sending $a_{i}$ to $a_{i+1}$ and $b_{i}$ to $b_{i+1}$. This extends to a free action of $\mathbb{Z} / 4$ on the eight octagons of $Y$. We define the involution $\tau$ to be the action of the element $2 \in \mathbb{Z} / 4$, so that $\tau . a_{i}=a_{i+2}$ and $\tau . b_{i}=b_{i+2}$. The fixed point set for the action of $\tau$ on $Y$ is just the central vertex of the rose, and so is a single point as claimed. Let $p: Y \rightarrow Y / \tau$ be the quotient map. There is a natural cell structure on $Y / \tau$ with one 0 -cell and four 1- and 2-cells. Since $p\left(a_{i}\right)=p\left(a_{i+2}\right)$ and $p\left(b_{i}\right)=p\left(b_{i+2}\right)$, the words describing the attaching maps for the four 2-cells are no longer reduced. After reduction they are equal to $p\left(a_{1}\right), p\left(a_{2}\right), p\left(b_{1}\right)$ and $p\left(b_{2}\right)$. Thus the 2-complex $Y / \tau$ is homotopy equivalent to a wedge of four 2-discs, and so is contractible.

The subcomplex of the rose consisting of the base vertex and the petals labelled $a_{0}$ and $a_{2}$ is a 2-petalled $\tau$-invariant rose which is isometrically embedded in $Y$.

REMARK 3.4. Here are two other families of group presentations that can be shown to arise as fundamental groups of finite locally $\mathrm{CAT}(0)$ square complexes using similar techniques:

- Generators $a, \ldots, f$, and relators

$$
\begin{gathered}
a b c b^{-1} e b, \quad a d c d^{-1} e d, \quad a f c f^{-1} e f \\
a b^{-1} c b^{2} e b^{-1}, \quad a^{2} d^{-1} c d e d^{-1}, \quad a f^{-1} c^{2} f e f^{-1} .
\end{gathered}
$$

- Fix $n \geq 8$, and take generators $a_{i}$ and relators $a_{i} a_{i+1} a_{i+3}^{-1} a_{i+1}^{-1} a_{i+3}$, for $i \in \mathbb{Z} / n$.

\section{Contractible and acyclic complexes}

For our main result we require a pair of locally $\mathrm{CAT}(0)$ cubical complexes $\left(A^{\prime}, A\right)$ and a cubical involution $\tau$ of the pair with the following properties:

(1) $A$ and $A^{\prime}$ are acyclic

(2) $A$ is not contractible

(3) $A$ is a totally geodesic subcomplex of $A^{\prime}$

(4) $A$ is contained in the fixed point set for $\tau$ acting on $A^{\prime}$

(5) $A^{\prime} / \tau$ is contractible

The following proposition gives a fairly simple construction that has almost all of the properties that we require.

Proposition 4.1. Let $A$ be an acyclic $C W$-complex, and let $\tau$ denote the involution of $A \times A$ defined by $\tau(a, b)=(b, a)$. The quotient space $C=(A \times A) /\langle\tau\rangle$ is contractible.

Proof. It suffices to show that the fundamental group $\pi_{1}(C)$ is trivial and that $C$ is acyclic. Since $\tau$ fixes points on the diagonal of $A \times A, \pi_{1}(C)$ is isomorphic to the quotient of the wreath product $\pi_{1}(A)$ wr $\langle\tau\rangle$ by the normal subgroup generated by $\tau$. Thus we need to show that this wreath product is generated by conjugates of $\tau$. Recall that elements of the wreath product may be written in the form $\left(\alpha, \beta, \tau^{\epsilon}\right)$, where $\alpha, \beta \in \pi_{1}(A)$ and $\epsilon=0$ or 1 . The product of two such elements is given by

$$
(\alpha, \beta, 1)\left(\gamma, \delta, \tau^{\epsilon}\right)=\left(\alpha \gamma, \beta \delta, \tau^{\epsilon}\right), \quad(\alpha, \beta, \tau)\left(\gamma, \delta, \tau^{\epsilon}\right)=\left(\alpha \delta, \beta \gamma, \tau^{1-\epsilon}\right)
$$


The inverse of $(\alpha, 1,1)$ is $\left(\alpha^{-1}, 1,1\right)$, and so for any $\alpha$,

$$
\left(\alpha, \alpha^{-1}, \tau\right)=(\alpha, 1,1)(1,1, \tau)\left(\alpha^{-1}, 1,1\right)
$$

is a conjugate of $\tau$, and $\left(\alpha, \alpha^{-1}, 1\right)$ is a product of conjugates of $\tau$. In particular, for any $\beta$ and $\gamma$,

$$
\left(\beta^{-1} \gamma^{-1} \beta \gamma, 1,1\right)=\left(\beta^{-1}, \beta, 1\right)\left(\gamma^{-1} \beta, \beta^{-1} \gamma, 1\right)\left(\gamma, \gamma^{-1}, 1\right)
$$

lies in the normal subgroup generated by $\tau$. But since $\pi_{1}(A)$ is perfect, an arbitary element of $\pi_{1}(A)$ may be expressed as a product of such commutators. Hence for any $\alpha,(\alpha, 1,1)$ and $(1, \alpha, 1)=(1,1, \tau)(\alpha, 1,1)(1,1, \tau)$ lie in the normal subgroup generated by $\tau$, which is therefore the whole of $\pi_{1}(A) \mathrm{wr}\langle\tau\rangle$.

Now let $\Delta$ denote the diagonal copy of $A$ within $A \times A$. Note also that $\Delta$ is equal to the fixed point set for the action of $\tau$ on $A \times A$. Since $A$ is acyclic, the inclusion of $\Delta$ in $A \times A$ induces a homology isomorphism. Hence the relative homology groups $H_{*}(A \times A, \Delta)$ are all trivial. It follows that the relative cellular chain complex $C_{*}(A \times A, \Delta)$ is an exact sequence of free $\mathbb{Z}\langle\tau\rangle$-modules, and hence is split. Thus this sequence remains exact upon tensoring over $\mathbb{Z}\langle\tau\rangle$ with $\mathbb{Z}$ (with $\tau$ acting trivially). Now there is a natural isomorphism

$$
C_{*}(C, \Delta)=C_{*}((A \times A) /\langle\tau\rangle, \Delta) \cong C_{*}(A \times A, \Delta) \otimes_{\mathbb{Z}\langle\tau\rangle} \mathbb{Z},
$$

and hence the relative homology groups $H_{*}(C, \Delta)$ are trivial. From this it follows that the inclusion of $\Delta$ in $C$ is a homology isomorphism, and hence that $C$ is acyclic, as required.

REMARK 4.2. For any prime $p$, a similar argument may be used to show that $A^{p} / C_{p}$ is contractible, where $C_{p}$ acts on $A^{p}$ by freely permuting the factors.

The only problem with the above construction is that if $A$ is an acyclic locally $\operatorname{CAT}(0)$ cubical complex, the diagonal copy of $A$ inside $A \times A$ is not a subcomplex. Nevertheless, the barycentric subdivision of the diagonal copy of $A$ is a subcomplex of the barycentric subdivision of $A \times A$.

Proposition 4.1 can be used to prove a weaker version of Theorem A, in which $T_{X}$ is not a locally CAT(0) cubical complex, but instead a locally CAT $(0)$ polyhedral complex. In the non-metric context, Proposition 4.1 is also useful. It can be used to give an alternative proof of the main theorem of [19], starting from a proof of the Kan-Thurston theorem along the lines of that of Baumslag-Dyer-Heller [3].

ThEOREM 4.3. There is a pair $\left(A^{\prime}, A\right)$ of locally $C A T(0)$ cubical complexes equipped with an involution $\tau$ having the properties listed at the start of this section. Furthermore $A$ is the fixed point set for the action of $\tau$. The complex $A$ is 2-dimensional and $A^{\prime}$ is 3-dimensional.

Proof. Let $Y$ be the 2-complex constructed in Proposition 3.3, and let $\tau^{\prime}$ denote the involution of $Y$ which is denoted ' $\tau$ ' in Proposition 3.3 . Let $X_{0}$ be the direct product $Y \times[-4,4]$ of $Y$ with an interval of length 8 , and define an involution $\tau_{0}$ on $X_{0}$ by $\tau_{0}(y, t)=\left(\tau^{\prime}(y),-t\right)$. By definition, $X_{0}$ can be viewed as being built from unit cubes. Define $X_{1}$ to be the mapping torus of $\tau^{\prime}: Y \rightarrow Y$, i.e., the quotient of $X_{0}$ obtained by identifying $(y, 4)$ and $\left(\tau^{\prime}(y),-4\right)$ for all $y \in Y$. Since $\tau^{\prime}$ is an involution, it follows readily that $\tau_{0}$ defines an involution $\tau_{1}$ on $X_{1}$. Let $y_{0}$ denote the unique 0 -cell in $Y$, and define $X_{2}$ by identifying the images of the points $\left(y_{0}, 0\right)$ and $\left(y_{0}, 4\right)$ in $X_{1}$. As before, $\tau_{1}$ passes to an involution $\tau_{2}$ on $X_{2}$. Each of $X_{0}, X_{1}$ and $X_{2}$ is a locally $\operatorname{CAT}(0)$ cubical complex, as may be seen by checking that all vertex links are flag. (Every vertex link in $X_{0}$ or $X_{1}$ is isomorphic either the cone on or the suspension of a 

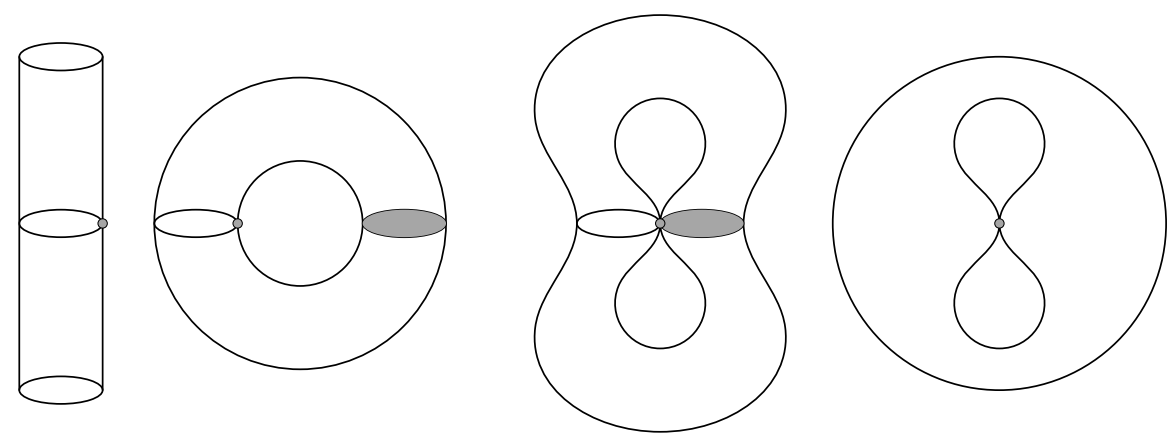

Figure 3 . The spaces $X_{0}, X_{1}, X_{2}$ and $X_{3}$, with the fixed point sets for the involutions shaded in grey.

vertex link in $Y$, and the 'new' link in $X_{2}$ is isomorphic to the disjoint union of two links from $X_{1}$.)

Let $Z_{2}$ be image in $X_{2}$ of $\left\{y_{0}\right\} \times[-4,4]$, so that $Z_{2}$ is a 2-petalled rose in which each petal has length 4 . The subspace $Z_{2}$ is totally geodesic and invariant under the action of $\tau$ (which swaps the two petals). Let $X_{3}$ be a disjoint copy of $Y$, and let $Z_{3}$ be the subspace of $X_{3}$ consisting of the 0-cell $y_{0}$ and the two 1-cells labelled $a_{1}$ and $a_{3}$. Then $Z_{3}$ is also a 2-petalled rose in which each petal has length $4, Z_{3}$ is totally geodesic in $Y$ and is invariant under the action of the involution $\tau^{\prime}$, which swaps the two petals. Let $\phi: Z_{2} \rightarrow Z_{3}$ be an isometry which is equivariant for the two given involutions, and define $A^{\prime}$ by taking the disjoint union of $X_{2}$ and $X_{3}$ and identifying $z$ with $\phi(z)$ for all $z \in Z_{2}$. Since $A^{\prime}$ was constructed from two locally CAT(0) cubical complexes by identifying isometric totally geodesic subcomplexes, it follows that $A^{\prime}$ is itself a locally $\mathrm{CAT}(0)$ cubical complex. (Alternatively, one may check the structure of vertex links in $A^{\prime}$.) Since $\phi$ has the property that $\phi \tau_{2}(z)=\tau^{\prime} \phi(z)$ for all $z \in Z_{2}$, one may define an involution $\tau$ on $A^{\prime}$ by $\tau(a)=\tau_{2}(a)$ for $a \in X_{2}$ and $\tau(a)=\tau^{\prime}(a)$ if $a \in X_{3}$. Let $A$ be the fixed point set for $\tau$. It is readily seen that $A$ is equal to the image of $Y \times\{0\}$ inside $A^{\prime}$, which is isometric to $Y$ and so is acyclic. The spaces $X_{0}, X_{1}, X_{2}$ and $X_{3}$ are depicted in Figure 3. The fixed point set for the involution on each $X_{i}$ is shaded in grey.

It remains to check that $A^{\prime}$ is acyclic and $A^{\prime} / \tau$ is contractible. Since $Y$ is acyclic, it follows easily that $X_{1}$ has the same homology as a circle. A Mayer-Vietoris argument now shows that the inclusion map $Z_{2} \rightarrow X_{2}$ is a homology isomorphism. Since $X_{3}$ is acyclic, another Mayer-Vietoris argument shows that $A^{\prime}$ is acyclic.

Note that $X_{1} / \tau_{1}$ is homeomorphic to the mapping cylinder of the quotient map $Y \rightarrow Y / \tau^{\prime}$. Since $Y / \tau^{\prime}$ is contractible, it follows that $X_{1} / \tau_{1}$ is contractible. From this it follows that $X_{2} / \tau_{2}$ is homotopy equivalent to a circle, and that the inclusion map $Z_{2} / \tau_{2} \rightarrow X_{2} / \tau_{2}$ is a homotopy equivalence. Since $X_{3} / \tau^{\prime}$ is contractible, it follows from van Kampen's theorem that $A^{\prime} / \tau^{\prime}$ has trivial fundamental group and it follows from the Mayer-Vietoris theorem that $A^{\prime} / \tau^{\prime}$ is acyclic. Hence $A^{\prime} / \tau^{\prime}$ is contractible as claimed.

\section{The main result}

Our proof is close to Maunder's proof of the Kan-Thurston theorem 22. However, we use a slightly wider class of spaces than Maunder's class of 'ordered simplicial complexes'. We start with the category of $\Delta$-complexes, or semi-simplicial sets [12, 30. Our construction will 
apply to the full subcategory $\mathcal{C}$ of $\Delta$-complexes such that the $\left(\begin{array}{c}n+1 \\ 2\end{array}\right)$ edges of each $n$-simplex are distinct.

Note that the barycentric subdivision of any $\Delta$-complex has the stronger property that the $(n+1)$ vertices of each $n$-simplex are distinct, and so the barycentric subdivision of any $\Delta$-complex is in $\mathcal{C}$. Similarly, the barycentric subdivision $K^{\prime}$ of any simplicial complex $K$ is naturally an object of $\mathcal{C}$, and any map $f: K \rightarrow L$ of simplicial complexes that is injective on each simplex induces a map of $\Delta$-complexes from $K^{\prime}$ to $L^{\prime}$.

Let $X$ and $Y$ denote $\Delta$-complexes, and let $x$ be a vertex of $X$. The link of $x$, denoted $\operatorname{Lk}_{X}(x)$, is another $\Delta$-complex. Any map of $\Delta$-complexes $f: X \rightarrow Y$ induces a map $f_{x}: \operatorname{Lk}_{X}(x) \rightarrow$ $\operatorname{Lk}_{Y}(f(x))$. (This is one advantage of $\Delta$-complexes over simplicial complexes.) The map $f$ is said to be locally injective if for each vertex $x$, the map $f_{x}$ is injective. It can be shown that a map of $\Delta$-complexes is locally injective if and only if the induced map of topological realizations is locally injective.

For each $X$ in $\mathcal{C}$, we will construct a locally CAT(0) cubical complex $T_{X}$, a map $t_{X}: T_{X} \rightarrow X$ and an involution $\tau$ on $T_{X}$ having the properties stated in Theorem A. The construction will be natural for any map $f: X \rightarrow Y$ in $\mathcal{C}$; however the induced map $T_{f}: T_{X} \rightarrow T_{Y}$ will be locally isometric only in the case when $f$ is locally injective. The following statement is a summary of what we will prove.

ThEOREM 5.1. Let $\mathcal{D}$ denote the category whose objects are locally $C A T(0)$ cubical complexes equipped with a cellular isometric involution $\tau$ and whose morphisms are $\tau$ equivariant cubical maps. There is a functor $T$ from $\mathcal{C}$ to $\mathcal{D}$ which has all of the properties listed in Theorem A.

Theorem A follows from Theorem [5.1 by composing $T$ with the barycentric subdivision functor from the category of simplicial complexes and simplicial maps that are injective on each simplex to the category $\mathcal{C}$.

Before beginning the proof of Theorem 5.1 we need two more pieces of notation. If $f$ : $T \rightarrow U$ is a locally isometric cellular map of cubical complexes, and $n$ is a positive integer, let $M(n, f)$ denote the mapping cylinder of $f$ of length $n$, i.e., the cubical complex obtained from $T \times[0, n] \amalg U$ by identifying, for each $t \in T$, the points $(t, n)$ and $f(t)$.

Finally, let $\left(A^{\prime}, A\right)$ be the pair of spaces constructed in Theorem 4.3. and let $j$ denote a fixed locally isometric closed loop $j:[0,4] \rightarrow A$. For example, $j$ could be the map that goes at constant speed around the generator $a_{1}$ for the fundamental group of $A$. (Recall that $A$ is isometric to the cubical complex $Y$ of Proposition 3.3])

Proof. For each finite $\Delta$-complex $X$ in $\mathcal{C}$, we inductively define $T_{X}$, the map $t_{X}: T_{X} \rightarrow X$, another locally CAT(0) cubical complex $U_{X}$, a locally isometric cubical map $i_{X}: T_{X} \rightarrow U_{X}$ and cubical involutions $\tau$ on $T_{X}, U_{X}$, so that all of the following hold.

- The map $t_{X}$ induces integral homology isomorphisms $T_{X} \rightarrow X$ and $T_{X}^{\tau} \rightarrow X$ and for any vertex $x_{0}$ of $X$, an isomorphism of fundamental groups $\pi_{1}\left(T_{X} /\langle\tau\rangle, T_{x_{0}}\right) \rightarrow \pi_{1}\left(X, x_{0}\right)$.

- $U_{X}$ is acyclic and $U_{X} / \tau$ is contractible.

- The map $i_{X}$ is $\tau$-equivariant.

- $T_{X}$ and $U_{X}$ are finite.

- $\operatorname{dim}\left(T_{X}\right)=\operatorname{dim}(X)$, except that if $\operatorname{dim}(X)=2$ then $\operatorname{dim}\left(T_{X}\right)=3$.

$-\operatorname{dim}\left(U_{X}\right)=\operatorname{dim}(X)+1$ except that $\operatorname{dim}\left(U_{X}\right)=3$ if $\operatorname{dim}(X)=1$, and $\operatorname{dim}\left(U_{X}\right)=0$ if $X$ is 0 -dimensional.

- If $W$ is a subcomplex of $X$, then $T_{W}$ is a totally geodesic subcomplex of $T_{X}$ and $U_{W}$ is a totally geodesic subcomplex of $U_{X}$, and these inclusions are $\tau$-equivariant. 
In the case when $X$ is 0 -dimensional, define $T_{X}$ to be $X$, define $U_{X}$ to be a single point, take $i_{X}: T_{X} \rightarrow U_{X}$ to be the unique map, and let $\tau$ be the trivial involution.

In the case when $X$ is 1-dimensional, take $T_{X}$ to be the second barycentric subdivision of $X$, so that for each edge $x$ of $X, T_{X}$ is either a loop or a path consisting of 4 edges of length 1. Let $\left(A^{\prime}, A\right)$ be the pair of spaces constructed in Theorem 4.3 and for $U_{X}$ take the 1-point union of copies of the space $A^{\prime}$ indexed by the 1-cells of $X$, with the involution $\tau$ as defined in Theorem4.3 The map $i_{X}: T_{X} \rightarrow U_{X}$ is defined by taking a copy of the map $j:[0,4] \rightarrow A \subseteq A^{\prime}$ for each 1-cell.

Now suppose that $T_{X}, U_{X}, \tau$ and $i_{X}$ have been defined and have the above listed properties whenever $X \in \mathcal{C}$ is finite and consists of at most $N-1$ simplices of various dimensions. Suppose now that $X$ is obtained from $W$ by adding a single $n$-simplex $\sigma$, for some $n \geq 2$. Let $\partial \sigma$ denote the subcomplex of $W$ consisting of all the simplices contained in the boundary of $\sigma$. To simplify notation slightly, let $U^{\prime}$ denote $U_{\partial \sigma}$, let $T^{\prime}$ denote the $\tau$-fixed point set $T_{\partial \sigma}^{\tau}$, and let $i^{\prime}: T^{\prime} \rightarrow U^{\prime}$ denote the restriction to $T^{\prime}$ of the map $i_{\partial \sigma}$. Now define

$$
\begin{gathered}
T_{X}=T_{W} \cup_{T^{\prime}} M\left(4, i^{\prime}: T^{\prime} \rightarrow U^{\prime}\right), \\
U_{X}=U_{W} \cup_{T^{\prime}}\left(T^{\prime} \times A\right) \cup_{A} A^{\prime} .
\end{gathered}
$$

More precisely, let $x_{0}$ be the initial vertex of $\sigma$, and let $w_{0}=T_{x_{0}}$ be the corresponding vertex of $T_{\sigma}$. Note that $w_{0}$ is fixed by the involution $\tau$ on $T_{\sigma}$. Also let $a_{0} \in A$ be the fixed point for the involution of $A$ described in Proposition 3.3. Now the space $U_{X}$ is obtained from the disjoint union $U_{X}=U_{W} \amalg\left(T^{\prime} \times A\right) \amalg A^{\prime}$ by identifying $T^{\prime} \times\left\{a_{0}\right\} \subseteq T^{\prime} \times A$ with $T^{\prime} \subseteq U_{W}$ and identifying $\left\{w_{0}\right\} \times A$ with $A \subseteq A^{\prime}$.

The involution $\tau$ on each of $T_{X}$ and $U_{X}$ is defined using the given involutions on $T_{W}, U_{W}$, $T^{\prime}, U^{\prime}$ and $A^{\prime}$. The map $i_{X}: T_{X} \rightarrow U_{X}$ is defined to equal $i_{W}$ on $T_{W}$. On $U^{\prime} \subseteq M\left(4, i^{\prime}\right)$ the map is equal to $i_{\partial \sigma}$, and on the rest of the mapping cylinder $M\left(4, i^{\prime}\right)$ it is induced by the map $(s, t) \mapsto(t, j(s))$ from $[0,4] \times T^{\prime}$ to $T^{\prime} \times A$. The map $t_{X}$ is defined by $t_{W}$ on $T_{W} \subseteq T_{X}$, by taking $U^{\prime} \subseteq M\left(4, i^{\prime}\right)$ to the barycentre of $\sigma$, and on the rest of the mapping cylinder $M(4, i)$ by linear interpolation between the map $\left.t_{W}\right|_{T^{\prime}}$ and the constant map that sends $T^{\prime}$ to the barycentre of $\sigma$.

It follows easily that the maps $T_{W} \rightarrow T_{X}$ and $U_{W} \rightarrow U_{X}$ are isometric embeddings of totally geodesic subcomplexes. The quickest way to see this is by induction, using a gluing lemma, but one could also show directly that each vertex link in $T_{W}$ (resp. $U_{W}$ ) is a full subcomplex of the corresponding vertex link in $T_{X}$ (resp. $U_{X}$ ). Similarly, an induction shows that the map $i_{X}: T_{X} \rightarrow U_{X}$ is a $\tau$-equivariant locally isometric cubical map.

It is easily checked that the map $t_{X}$ induces isomorphisms from each of $H_{*}\left(T_{X}, T_{W}\right)$, $H_{*}\left(T_{X}^{\tau}, T_{W}^{\tau}\right)$ and $H_{*}\left(T_{X} / \tau, T_{W} / \tau\right)$ to $H_{*}(X, W)$. By the 5 -lemma and induction it follows that $t_{X}$ induces isomorphisms from $H_{*}\left(T_{X}\right), H_{*}\left(T_{X}^{\tau}\right)$ and $H_{*}\left(T_{X} / \tau\right)$ to $H_{*}(X)$.

By induction, the fundamental group of $U^{\prime} / \tau$ is trivial, and $t_{W}$ induces an isomorphism $\pi_{1}\left(T_{W} / \tau, T_{w_{0}}\right) \rightarrow \pi_{1}\left(W, w_{0}\right)$, for any vertex $w_{0} \in W$. By Van Kampen's theorem, it follows that $t_{W}$ induces an isomorphism from $\pi_{1}\left(T_{X} / \tau, T_{x_{0}}\right)$ to $\pi_{1}\left(X, x_{0}\right)$ as claimed.

The Mayer-Vietoris theorem shows that $H_{*}\left(U_{X}, U_{W}\right)=\{0\}$, and so by induction $U_{X}$ is acyclic. Similarly, Van Kampen's theorem and an induction shows that $U_{X}$ is simply-connected. It follows that $U_{X}$ is contractible as required. This completes the proof that the construction applies to any finite $X$ and has the properties listed at the start of the proof in this case.

For the general case, define $T_{X}, t_{X}$ and $\tau$ as direct limits over the finite subcomplexes of $X$. (We shall not need $U_{X}$ or $i_{X}$ in the case when $X$ is infinite, but these could also be defined in this way for arbitrary $X$.) It is immediate from the definition that in the general case, $t_{X}$ induces isomorphisms from $H_{*}\left(T_{X}\right), H_{*}\left(T_{X}^{\tau}\right)$ and $H_{*}\left(T_{X} / \tau\right)$ to $H_{*}(X)$ and an isomorphism, for any $x_{0}$, from $\pi_{1}\left(T_{X}, T_{x_{0}}\right)$ to $\pi_{1}\left(X, x_{0}\right)$. 
To establish the metric properties of $T_{X}$ in the general case, it is easiest to use Gromov's criterion (Theorem B.8). If $t$ is a vertex of $T_{X}$, then the link of $t, \operatorname{Lk}_{T_{X}}(t)$, is the limit of the $\mathrm{Lk}_{T_{Y}}(t)$, where $Y$ ranges over the finite subcomplexes of $X$ such that $T_{Y}$ contains $t$. Since each such $\operatorname{Lk}_{T_{Y}}(t)$ is a flag complex, it follows that $\operatorname{Lk}_{T_{X}}(t)$ is a flag complex. Hence by Theorem B.8, $T_{X}$ is locally CAT(0). Now suppose that $W$ is a subcomplex of $X$, let $t$ be a vertex of $T_{W}$, and let $e_{0}, \ldots, e_{n}$ be vertices of $\operatorname{Lk}_{T_{W}}(t)$. Let $U$ be any finite subcomplex of $W$ such that $t$ is a vertex of $T_{U}$ and such that each of $e_{0}, \ldots, e_{n}$ is a vertex of $\operatorname{Lk}_{T_{U}}(t)$. If $Y$ is any finite subcomplex of $X$ that contains $U$, then $\operatorname{Lk}_{T_{U}}(t)$ is a full subcomplex of $\operatorname{Lk}_{T_{Y}}(t)$. It follows that every simplex of $\operatorname{Lk}_{T_{X}}(t)$ whose vertex set is contained in $\left\{e_{0}, \ldots, e_{n}\right\}$ is already a simplex in $\operatorname{Lk}_{T_{U}}(t)$. Hence $\operatorname{Lk}_{T_{W}}(t)$ is a full subcomplex of $\operatorname{Lk}_{T_{X}}(t)$, and so the inclusion $T_{W} \rightarrow T_{X}$ is totally geodesic. This completes the construction of $T_{X}$ and $t_{X}$ having all the properties listed at the start of the proof.

To complete the proof of Theorem [5.1, it remains to establish that $T_{X}, t_{X}$ and $\tau$ have the rest of the properties listed in the statement of Theorem A. We first consider the three listed naturality properties. The fact that any map of $\Delta$-complexes $f: X \rightarrow Y$ induces a map of cubical complexes $T_{f}: T_{X} \rightarrow T_{Y}$ is clear from the construction. The fact that $T_{f}$ embeds $T_{X}$ isometrically as a totally geodesic subcomplex in the case when $f$ is injective follows immediately from the case when $f$ is the inclusion of a subcomplex, which was proved during the construction of $T_{X}$. The fact that $T_{f}$ is locally isometric when $f$ is locally injective now follows from Theorem B.9. since if $f$ is locally injective then for any vertex $t$ of $T_{X}$, the induced map from $\operatorname{Lk}_{T_{X}}(t)$ to $\operatorname{Lk}_{T_{Y}}(f(t))$ is the inclusion of a full subcomplex in a flag complex.

In the case when $X$ is simply-connected, properties 1-3 listed in the statement of Theorem A follow from the properties that we have already established. In the case when $X$ is connected, but not simply connected, let $\widetilde{X}$ be the universal cover of $X$, and let $G$ be the fundamental group of $X$. By naturality of the construction, $G$ acts by deck transformations on $T_{\widetilde{X}}$. The covering map $\widetilde{X} \rightarrow X$ induces a map $T_{\widetilde{X}} \rightarrow T_{X}$, which commutes with the action of $G$ on $T_{\widetilde{X}}$. It follows that there is a $G$-equivariant isomorphism from $T_{\widetilde{X}}$ to the regular cover of $T_{X}$ with fundamental group equal to the kernel of the map from $\pi_{1}\left(T_{X}\right)$ to $G$. Note also that the map $T_{\widetilde{X}} \rightarrow \widetilde{X}$ induces a $G$-equivariant homotopy equivalence $T_{\widetilde{X}} / \tau \rightarrow \widetilde{X}$. It follows that in the case when $X$ is connected, the map $T_{X} / \tau \rightarrow X$ is a homotopy equivalence. For each $n \geq 0$, let $\widetilde{X}^{n}$ denote the $n$-skeleton of the universal cover of $X$, and let $\widetilde{X}^{-1}$ denote the empty space. By what we have proved already, for each $n \geq-1$ the map induced by $t_{X}$ from $H_{*}\left(T_{\widetilde{X}^{n+1}}, T_{\widetilde{X}^{n}}\right)$ to $H_{*}\left(X^{n+1}, X^{n}\right)$ is a $G$-equivariant isomorphism. It follows by induction that for any local coefficient system $M$ on $X$ and for any $n \geq 0$, the map $t$ induces an isomorphism $H_{*}\left(T_{X^{n}} ; M\right) \rightarrow H_{*}\left(X^{n} ; M\right)$. This establishes property 1 in the case when $X$ is connected and finite-dimensional. The case when $X$ is connected follows from this case, since the inclusion of $X^{n}$ into $X$ induces an isomorphism on $H_{i}(-; M)$ for any $i<n$.

The proof of property 3 in the case when $X$ is connected is very similar to the proof of property 1 in the connected case. Properties $1-3$ in the case of general $X$ follow immediately from the connected case.

\section{Some other properties of $T_{X}$}

Theorem 6.1. For any odd prime $p$, there is a cubical complex $T_{p, X}$ which has similar properties to those described in the statement of Theorem A, except that the involution $\tau$ of $T_{X}$ is replaced by an order $p$ cellular isometry $\tau_{p}$ of $T_{p, X}$.

Proof. It suffices to prove the $p$-analogue of Theorem [5.1. The given proof of Theorem 5.1] will apply, provided that we establish a suitable $p$-analogue of Theorem 4.3 . For this we need 
a $p$-analogue of Proposition 3.3. In fact, if one puts $n=2 p$ and uses the $2 n$ words described exactly as in the proof of Proposition 3.3. one obtains an acyclic 2-complex $Y_{p}$ consisting of $4 p$ octagons attached to a $4 p$-petalled rose and admitting a free $\mathbb{Z} / 2 p$-action. If $\tau_{p}$ is an order $p$ element of $\mathbb{Z} / 2 p$, then the action of $\tau_{p}$ on $Y_{p}$ has the required properties.

The $p$-analogue of Theorem 4.3 is fairly similar to the original, but the definition of $X_{1, p}$, the $p$-analogue of the space $X_{1}$ appearing in the proof, may require some clarification. Let $Y_{p}^{\prime}$ denote a second copy of $Y_{p}$, but with the trivial $\mathbb{Z} / p$-action instead of the action described above, and let $Y_{p}^{\prime \prime}$ be a disjoint union of $p$ copies of $Y_{p}^{\prime}$, with $\mathbb{Z} / p$ acting by freely permuting the copies. (One could view $Y_{p}^{\prime \prime}$ as the direct product $Y_{p}^{\prime} \times \mathbb{Z} / p$ with the diagonal action of $\mathbb{Z} / p$.) There are two $\mathbb{Z} / p$-equivariant covering maps $f: Y_{p}^{\prime \prime} \rightarrow Y_{p}^{\prime}$ and $g: Y_{p}^{\prime \prime} \rightarrow Y_{p}$. The $\mathbb{Z} / p$-space $X_{1, p}$ is defined to be the double mapping cylinder of $f$ and $g$ :

$$
X_{1, p}=\left(Y^{\prime} \amalg Y^{\prime \prime} \times[0,4] \amalg Y\right) /(y, 0) \sim f(y), \quad(y, 4) \sim g(y) \quad \text { for all } y \in Y^{\prime \prime} .
$$

The remainder of the proof carries over easily to provide a pair $\left(A_{p}^{\prime}, A_{p}\right)$ of locally $\operatorname{CAT}(0)$ cubical complexes with the required properties.

Proposition 6.2. The cubical complex $T_{X}$ is the cubical subdivision of a cube complex $\bar{T}_{X}$ which has similar properties.

Proof. This involves checking that each of the cubical complexes used in the construction can be viewed as the cubical subdivision of a cube complex. The octagons used in the construction of $Y$ in Proposition 3.3 can be chosen to be cubical subdivisions, in which case $Y$ is the cubical subdivision of a cube complex $\bar{Y}$. From this it follows that the cubical complexes $A$ and $A^{\prime}$ constructed in Theorem 4.3 are the cubical subdivisions of cube complexes $\bar{A}$ and $\bar{A}^{\prime}$. Now a cube complex $\bar{T}_{X}$ whose cubical subdivision is $T_{X}$ can be constructed from $\bar{A}$ and $\bar{A}^{\prime}$ exactly as in the proof of Theorem 5.1 .

Proposition 6.3. For any $X$, the map $t_{X}: T_{X} \rightarrow X$ has the property that for any subcomplex $Y$ of $X, t_{X}^{-1}(Y)=T_{Y}$. In particular, the map $t_{X}$ is proper.

Proof. Immediate from the construction.

Proposition 6.4. The locally CAT(0) cubical complex $T_{X}$ is metrically complete if and only if $X$ has no infinite ascending chain of simplices.

Proof. By Theorem A.6, a locally CAT(0) cubical complex is metrically complete if and only if it contains no infinite ascending chain of cubes. It is immediate from the construction that this condition holds for $T_{X}$ if and only if $X$ contains no infinite ascending chain of simplices. (Equivalently, again by Theorem A.6. $T_{X}$ is complete if and only if the all-right metric on $X$ is complete.)

Proposition 6.5. There is a locally $C A T(0)$ cubical complex $T_{X}^{\prime}$, equipped with an involution $\tau^{\prime}$ and a map $t_{X}^{\prime}: T_{X}^{\prime} \rightarrow X$ which has all of the properties listed in Theorem [5.1. and such that $T_{X}^{\prime}$ is metrically complete for every $X$. The map $t_{X}^{\prime}: T_{X}^{\prime} \rightarrow X$ is proper if and only if $X$ is finite-dimensional. 
Proof. If $X$ is $n$-dimensional, define $T_{X}^{\prime}$ to be the iterated mapping cylinder of the inclusion maps

$$
T_{X^{0}} \rightarrow T_{X^{1}} \rightarrow \cdots \rightarrow T_{X^{n}}
$$

From the definition of this space comes a map $p_{X}: T_{X}^{\prime} \rightarrow T_{X}$. In general, define $T_{X}^{\prime}$ to be the union of the subspaces $T_{X^{n}}^{\prime}$, and define a map $p_{X}: T_{X}^{\prime} \rightarrow T_{X}$ as the direct limit of the maps $p_{X^{n}}$. Now define $t_{X}^{\prime}=t_{X} \circ p_{X}$. The claimed properties follow readily from these definitions.

REMark 6.6. If $x_{0}$ is a vertex of $X$, then $x_{0}^{\prime}=T_{x_{0}}$ is a vertex of $T_{X}$ which is fixed by the $\tau$-action. Thus $t_{X}$ induces maps of fundamental groups

$$
t_{X *}: \pi_{1}\left(T_{X}, x_{0}^{\prime}\right) \rightarrow \pi_{1}\left(X, x_{0}\right), \quad t_{X *}: \pi_{1}\left(T_{X}^{\tau}, x_{0}^{\prime}\right) \rightarrow \pi_{1}\left(X, x_{0}\right),
$$

which are easily seen to be surjective. Note also that $\tau$ induces an automorphism $\tau_{*}$ of $\pi_{1}\left(T_{X}, x_{0}^{\prime}\right)$. Since $T_{X}^{\tau}$ includes into $T_{X}$ as a totally geodesic subcomplex, it follows that $\pi_{1}\left(T_{X}^{\tau}, x_{0}^{\prime}\right)$ is equal to the centralizer of $\tau_{*}$ in $\pi_{1}\left(T_{X}, x_{0}^{\prime}\right)$.

\section{Arbitrary spaces}

If $X$ is any topological space, then the first barycentric subdivision of the space of singular simplicies, $\operatorname{Sing}(X)$, is in the category $\mathcal{C}$ (the category of $\Delta$-complexes in which all the edges of each simplex are distinct). Composing this functor with our construction $T$ gives the following result.

Theorem 7.1. There is a functor $\widehat{T}$ from topological spaces to the category $\mathcal{D}$ as defined in Theorem 5.1, an involution $\tau$ of $\widehat{T}_{X}$ and a map $\widehat{t}_{X}: \widehat{T}_{X} \rightarrow X$ such that

(i) The map $\hat{t}_{X}$ induces an isomorphism on singular homology for any local coefficients on $X$.

(ii) The involution $\tau$ on $\widehat{T}_{X}$ has the property that $\widehat{t}_{X} \circ \tau=\widehat{t}_{X}$ and the induced map $\widehat{T}_{X} /\langle\tau\rangle \rightarrow X$ is a weak homotopy equivalence.

(iii) The map $\widehat{t}_{X}: \widehat{T}_{X}^{\tau} \rightarrow X$ induces an isomorphism on singular homology for any local coefficients on $X$, where $\widehat{T}_{X}^{\tau}$ denotes the fixed point set in $\widehat{T}_{X}$ for the action of $\tau$.

(iv) A continuous map $f: X \rightarrow Y$ gives rise to a cubical map $\widehat{T}_{f}: \widehat{T}_{X} \rightarrow \widehat{T}_{Y}$.

(v) If $f$ is injective, then $\widehat{T}_{f}$ embeds $\widehat{T}_{X}$ as a totally geodesic subcomplex of $\widehat{T}_{Y}$.

(vi) If $f$ is locally injective, then $\widehat{T}_{f}$ is a locally isometric map.

REMARK 7.2. The theorem requires no connectivity assumptions on $X$ provided that singular homology with local coefficients is defined as in [33, Ch. VI.2].

Proof. The only point that needs some verification is that a locally injective map of topological spaces $f: X \rightarrow Y$ induces a locally injective map of $\Delta$-complexes $\operatorname{Sing}(f)$ : $\operatorname{Sing}(X) \rightarrow \operatorname{Sing}(Y)$.

\section{Generalized and equivariant homologies}

Let $\mathcal{K}$ denote any generalized homology theory satisfying the direct sum axiom (equivalently, the corresponding reduced theory satisfies Milnor's wedge axiom). A standard argument gives: 
Proposition 8.1. For any $X \in \mathcal{C}$ and any $\mathcal{K}$, the map $t_{X}$ induces an isomorphism from $\mathcal{K}_{*}\left(T_{X}\right)$ to $\mathcal{K}_{*}(X)$.

Proof. Prove also the corresponding statement for relative homology. First consider the case of a pair $(X, Y)$, where $X$ is $n$-dimensional and $Y$ is a subcomplex of $X$ so that $X-Y$ consists of a single $n$-simplex. In this case the Atiyah-Hirzebruch spectral sequence for computing the relative groups $\mathcal{K}_{*}\left(T_{X}, T_{Y}\right)$ collapses, and by naturality of this spectral sequence one sees that the map $t_{X}:\left(T_{X}, T_{Y}\right) \rightarrow(X, Y)$ induces an isomorphism $\mathcal{K}_{*}\left(T_{X}, T_{Y}\right) \rightarrow \mathcal{K}_{*}(X, Y)$. The case when $X=X^{n}$ is $n$-dimensional and $Y$ is the $(n-1)$-skeleton of $X$ follows (here we use the direct sum axiom).

For finite-dimensional $X$, the absolute case follows from the relative case by induction. The general case follows from this, since (again using the direct sum axiom) one knows that the natural map from the direct limit of the $\mathcal{K}_{*}\left(X^{n}\right)$ to $\mathcal{K}_{*}(X)$ is an isomorphism, and similarly the natural map from the direct limit of the $\mathcal{K}_{*}\left(T_{X^{n}}\right)$ to $\mathcal{K}_{*}\left(T_{X}\right)$ is an isomorphism. The general relative case now follows.

Now suppose that $G$ is a group acting on $X \in \mathcal{C}$, and that $\mathcal{K}_{*}^{G}$ is a $G$-homology theory in the sense of [20]. (Roughly speaking, this is a sequence of functors from pairs of $G$-CW-complexes to abelian groups which satisfies analogues of the Eilenberg-Steenrod axioms (except the dimension axiom) and a direct sum axiom.) Then $G$ also acts on $T_{X}$, and a mild generalization of the argument given above gives:

Proposition 8.2. For any such $X, G$ and $\mathcal{K}_{*}^{G}$, the map $t_{X}: T_{X} \rightarrow X$ is $G$-equivariant and induces an isomorphism $\mathcal{K}_{*}^{G}\left(T_{X}\right) \rightarrow \mathcal{K}_{*}^{G}(X)$.

Before continuing, we make some remarks concerning equivariant homology theories. Lück has given two slightly different definitions of an equivariant homology theory $\mathcal{K}_{*}^{\text {? }}$, one more restrictive than the other. The results of this section are valid for the less restrictive definition, but we shall require the more restrictive definition in Section 10, and so we briefly describe both. The less restrictive definition is given in [20, sec. 1]. The more restrictive definition is given in [21, def. 4.2]. The special case of [1, def. 1.3] (a definition of an 'equivariant homology theory over a group $\Gamma^{\prime}$ ') in which the group $\Gamma$ is trivial is equivalent to [21, def. 4.2].

In each case we are given, for each group $G$ a $G$-homology theory $\mathcal{K}_{*}^{G}$, together with induction maps that relate these theories for different groups. If $\alpha: H \rightarrow G$ is a group homomorphism and $X$ is an $H$-CW-complex, there is a $G$-CW-complex $\operatorname{ind}_{\alpha}(X)$, defined as the quotient space:

$$
G \times X /(g, x) \sim\left(g \alpha(h), h^{-1} x\right) \quad \text { for all } h \in H .
$$

In the more restrictive definition, there is a map $\operatorname{ind}_{\alpha}: \mathcal{K}_{*}^{H}(X) \rightarrow \mathcal{K}_{*}^{G}(X)$ for every $H$-CWcomplex $X$ and every group homomorphism $\alpha$. Moreover, the map ind ${ }_{\alpha}$ is required to be an isomorphism whenever $\operatorname{ker}(\alpha)$ acts freely on $X$. In the less restrictive definition, the map $\operatorname{ind}_{\alpha}$ is only required to be defined in the case when $\operatorname{ker}(\alpha)$ acts freely on $X$ (when it is still an isomorphism).

For the remainder of this section, we assume that $\mathcal{K}_{*}^{?}$ is an equivariant homology theory in the sense of [20]. Before stating the main result of this section, we require one more definition. For $G$ a discrete group, a $G$-CW-complex $E$ is said to be a model for $\underline{\mathrm{E}} G$ if all stabilizer subgroups for $E$ are finite and if for every finite subgroup $K \leq G$, the fixed point set $E^{K}$ is contractible. It is easily shown that such an $E$ is unique up to equivariant homotopy equivalence. 
Theorem 8.3. Let $G$ act on $X \in \mathcal{C}$, so that the stabilizer of each simplex is finite. Assume also that $X / G$ is connected. There is a group $\widetilde{G}$, a group homomorphism $\alpha: \widetilde{G} \rightarrow G$ with $\operatorname{ker}(\alpha)$ torsion-free, and a $\widetilde{G}$-equivariant map $t: \underline{\mathrm{E}} \widetilde{G} \rightarrow X$ such that for any equivariant homology theory $\mathcal{K}_{*}^{?}$, the map

$$
t_{*}: \mathcal{K}_{*}^{\widetilde{G}}(\underline{\mathrm{E}} \widetilde{G}) \rightarrow \mathcal{K}_{*}^{G}(X)
$$

is an isomorphism. In the case when $X$ is connected, the homomorphism $\alpha$ is surjective.

Proof. First, suppose that $X$ is not connected. In this case pick $X_{0}$, a component of $X$, and let $G_{0}$ be the setwise stabilizer of $X_{0}$. If $\beta$ denotes the inclusion homomorphism $\beta: G_{0} \rightarrow$ $G$, then $X$ is equivariantly homeomorphic to $\operatorname{ind}_{\beta}\left(X_{0}\right)$, and $\operatorname{ind}_{\beta}: \mathcal{K}_{*}^{G_{0}}\left(X_{0}\right) \rightarrow \mathcal{K}_{*}^{G}(X)$ is an isomorphism. Hence it suffices to consider the case when $X=X_{0}$ is connected.

In the connected case, consider $t_{X}: T_{X} \rightarrow X$. By naturality the group $G$ acts as a group of automorphisms of $T_{X}$. Let $E$ be the universal covering space of $T_{X}$, define $t: E \rightarrow X$ to be the composite of the projection map $E \rightarrow T_{X}$ and $t_{X}: T_{X} \rightarrow X$, and let $\widetilde{G}$ be the group of all selfmaps of $E$ that lift the action of $G$ on $T_{X}$. By construction, there is a surjective homomorphism $\alpha: \widetilde{G} \rightarrow G$ with $\operatorname{ker}(\alpha)$ equal to the group of deck transformations of the covering $E \rightarrow T_{X}$. By the previous proposition, the map $\mathcal{K}_{*}^{G}\left(T_{X}\right) \rightarrow \mathcal{K}_{*}^{G}(X)$ is an isomorphism. Since the group of deck transformations acts freely on $E$, it follows that $\operatorname{ind}_{\alpha}(E) \cong E / \operatorname{ker}(\alpha)=T_{X}$ and that the map $\operatorname{ind}_{\alpha}: \mathcal{K}_{*}^{\widetilde{G}}(E) \rightarrow \mathcal{K}_{*}^{G}(X)$ is defined and is an isomorphism. But $E$ is a $\operatorname{CAT}(0)$ cubical complex, and $\widetilde{G}$ acts cellularly on $E$ with finite stabilizers. It follows from Theorem B.11 that $E$ is a model for $\underline{E} \widetilde{G}$. Thus if we put $t=t_{X} \circ \operatorname{ind}_{\alpha}: E \rightarrow X$ we get the claimed result.

\section{Borel equivariant cohomology}

Consider the Borel equivariant cohomology of a $G$-CW-complex $X$, defined as $H_{G}^{*}(X):=$ $H^{*}\left(E G \times_{G} X\right)$. Note that in the case when $X$ is contractible, this is just the cohomology $H^{*}(G)$ of the group $G$. In general, by the simplicial approximation theorem, we may assume that $X$ and the $G$-action on $X$ are in $\mathcal{C}$. If all stabilizers in $X$ are finite and $X / G$ is connected, then with the notation of Theorem 8.3 , we see that there is a chain of isomorphisms

$$
t^{*}: H_{G}^{*}(X) \rightarrow H_{\widetilde{G}}^{*}(\underline{\mathrm{E}} \widetilde{G}) \cong H^{*}(\widetilde{G}) .
$$

In particular, for any such $G$ and $X$, there is a group $\widetilde{G}$ for which the group cohomology ring $H^{*}(\widetilde{G})$ is isomorphic to the Borel equivariant cohomology $\operatorname{ring} H_{G}^{*}(X)$.

Let $p$ be a prime, and let $\mathbb{F}_{p}$ be the field of $p$ elements. In theorem 14.1 of [29], Quillen described, up to F-isomorphism, the cohomology ring $H^{*}\left(\Gamma ; \mathbb{F}_{p}\right)$ of any discrete group $\Gamma$ of finite virtual cohomological dimension over $\mathbb{F}_{p}$. In part I of [29], Quillen had already described, up to F-isomorphism, the Borel equivariant cohomology ring $H_{G}^{*}\left(X ; \mathbb{F}_{p}\right)$ for any finite $G$ and finitedimensional $G$-CW-complex $X$. The proof of theorem 14.1, contained in sections 15 and 16 of [29], involved exhibiting an isomorphism between $H^{*}\left(\Gamma ; \mathbb{F}_{p}\right)$ and $H_{G}^{*}(X)$ for some finite $G$ and suitable $X$. In the case when $\Gamma$ has finite virtual cohomological dimension over $\mathbb{Z}, G$ may be taken to be $\Gamma / N$ for any finite-index torsion-free normal subgroup $N$, and $X$ may be taken to be $\underline{\mathrm{E}} \Gamma / N$. (Note that a group of finite vcd over $\mathbb{Z}$ necessarily has finite vcd over $\mathbb{F}_{p}$, but the converse does not always hold.)

One corollary of Theorem 8.3 is that this process can be reversed: if we take as given Quillen's description of $H^{*}\left(\Gamma ; \mathbb{F}_{p}\right)$ for all groups $\Gamma$ of finite vcd over the integers, then we can deduce Quillen's description of the Borel equivariant cohomology ring $H_{G}^{*}\left(X ; \mathbb{F}_{p}\right)$ for all finite groups $G$ and all finite-dimensional $G$-CW-complexes $X$. Moreover, we can deduce a theorem not stated in 29 by similar methods. To justify this assertion, we need to define transport categories. 
Let $X$ be a $G$-CW-complex with all stabilizers finite. Following [29, we define the transport category $\mathcal{T}(G, X)$. The objects of $\mathcal{T}(G, X)$ are pairs $(H, c)$, where $H$ is a finite subgroup of $G$ and $c$ is a component of the $H$-fixed point set $X^{H}$. The morphisms from $(H, c)$ to $\left(H^{\prime}, c^{\prime}\right)$ are the group homomorphisms $H \rightarrow H^{\prime}$ of the form $h \mapsto g h g^{-1}$ for some $g \in G$ such that $g . c$ is contained in $c^{\prime}$. In the case when $X=\underline{\mathrm{E}} G$, the transport category $\mathcal{T}(G, \underline{\mathrm{E}} G)$ is isomorphic to the Frobenius category $\Phi(G)$. The objects of $\Phi(G)$ are the finite subgroups of $G$, with morphisms the group homomorphisms that are induced by inner automorphisms of $G$. For $p$ a prime, let $\mathcal{A}_{p}(G, X)$ denote the full subcategory of $\mathcal{T}(G, X)$ with objects the pairs $(A, c)$ such that $A$ is abelian of exponent $p$. Quillen's description of $H_{G}^{*}\left(X ; \mathbb{F}_{p}\right)$, for $G$ finite, is as an inverse limit over $\mathcal{A}_{p}(G, X)$.

Proposition 9.1. With notation as in the statement of Theorem 8.3, the map $t: \underline{\mathrm{E}} \widetilde{G} \rightarrow X$ induces an equivalence from the Frobenius category $\Phi(\widetilde{G})$ to the transport category $\overline{\mathcal{T}}(G, X)$.

Proof. Since the kernel of the map $\alpha: \widetilde{G} \rightarrow G$ is torsion-free, any finite subgroup of $\widetilde{G}$ maps isomorphically to a finite subgroup of $G$. Let $(H, c)$ be any object in $\mathcal{T}(G, X)$, let $T_{c}$ be the inverse image of $c$ in $T_{X}$, and let $c^{\prime}$ be any lift of $T_{c}$ to $E$, the universal cover of $T_{X}$. For any $x \in c^{\prime}$, there is a subgroup $H^{\prime}$ of $\widetilde{G}$ which fixes $x$ and maps isomorphically to $H$. If $c^{\prime \prime}$ is another lift of $c$, and $H^{\prime \prime}$ is defined similarly to $H^{\prime}$, then there is an element $g \in \widetilde{G}$ such that $g \cdot c^{\prime}=c^{\prime \prime}$ and $g H^{\prime} g^{-1}=H^{\prime \prime}$, and so $\left(H^{\prime}, c^{\prime}\right)$ is isomorphic to $\left(H^{\prime \prime}, c^{\prime \prime}\right)$ as objects of $\Phi(\widetilde{G})$.

Corollary 9.2. For any virtually torsion-free group $G$ and any finite-dimensional $G$ - $C W$ complex $X$ with finite stabilizer subgroups, the natural map

$$
H_{G}^{*}\left(X ; \mathbb{F}_{p}\right) \rightarrow \operatorname{inv}_{(A, c) \in \lim _{p}(G, X)} H^{*}\left(A ; \mathbb{F}_{p}\right)
$$

is a uniform F-isomorphism.

Proof. The statement easily reduces to the case when $X / G$ is connected. In this case, by Theorem 8.3 , there exists a discrete group $\widetilde{G}$, a homomorphism $\alpha: \widetilde{G} \rightarrow G$ and a $\widetilde{G}$-equivariant map $t: \underline{\mathrm{E}} \widetilde{G} \rightarrow X$ inducing an isomorphism from $H^{*}\left(\widetilde{G} ; \mathbb{F}_{p}\right)$ to $H_{G}^{*}\left(X ; \mathbb{F}_{p}\right)$. The map $t$ also induces an equivalence of categories from $\Phi(\widetilde{G})$ to $\mathcal{T}(G, X)$. Since $G$ is virtually torsion-free and $X$ is finite-dimensional, it follows that $\widetilde{G}$ is of finite virtual cohomological dimension. The result now follows from Theorem 14.1 of $[\mathbf{2 9}]$.

\section{Assembly conjectures}

A family $\mathcal{F}$ of subgroups of a group $G$ is a non-empty collection of subgroups which is closed under conjugation and taking subgroups, i.e., for any $H \in \mathcal{F}$, any $g \in G$ and any $K \leq H$, $g H g^{-1} \in \mathcal{F}$ and $K \in \mathcal{F}$. Examples include the family of all subgroups of $G$, the family of finite subgroups, and the family consisting of just the trivial subgroup.

A model for $E(\mathcal{F}, G)$, the classifying space for actions of $G$ with stabilizers in $\mathcal{F}$, is a $G$-CWcomplex $E$ such that each cell stabilizer is in $\mathcal{F}$ and such that for any $H \in \mathcal{F}$, the $H$-fixed point set $E^{H}$ is contractible. It can be shown that for any $G$ and $\mathcal{F}$ there exist models for $E(\mathcal{F}, G)$, and that for fixed $G$ and $\mathcal{F}$ any two such models are equivariantly homotopy equivalent. In the case when $\mathcal{F}$ is the family of all subgroups of $G$, a single point $*$ is a model for $E(\mathcal{F}, G)$. In the case when $\mathcal{F}$ is just the trivial subgroup, a model for $E(\mathcal{F}, G)$ is the same thing as a model for $E G$, the universal free $G$-CW-complex. In the case when $\mathcal{F}$ is the family of finite subgroups of $G$, a model for $E(\mathcal{F}, G)$ is the same thing as a model for $\underline{E} G$, as defined just 
above the statement of Theorem 8.3. If $G$ acts cellularly on a CAT(0) cubical complex in such a way that all cell stabilizers are finite, then from Theorem B.11 it follows that $X$ is a model for $\underline{E} G$.

Let $\mathcal{K}_{*}^{\text {? }}$ be an equivariant homology theory in the sense of [20, let $G$ be a group, and let $\mathcal{F}$ be a family of subgroups of $G$. The $\left(\mathcal{K}_{*}^{?}, \mathcal{F}, G\right)$ assembly conjecture states that the map $E(\mathcal{F}, G) \rightarrow *$ induces an isomorphism from $\mathcal{K}_{*}^{G}(E(\mathcal{F}, G))$ to $\mathcal{K}_{*}^{G}(*)$.

For example, if $\mathcal{F}$ is the family of all subgroups, then for any $\mathcal{K}_{*}^{?}$ and any group $G$, the assembly conjecture holds. This example illustrates the principle that assembly conjectures are stronger when they involve smaller families of groups. One way to look at an assembly conjecture is as saying that $\mathcal{K}_{*}^{G}(*)$ is determined by knowledge of $\mathcal{K}_{*}^{H}(*)$ for groups $H \in \mathcal{F}$, together with knowledge of how the $\mathcal{F}$-subgroups of $G$ fit together (embodied in the structure of $E(\mathcal{F}, G)$ ).

Another example of an assembly conjecture that holds for trivial reasons comes from Borel equivariant homology. For any contractible $G$-CW-complex $E$, one has that $E G \times E$ is a free, contractible $G$-CW-complex. Hence the assembly conjecture for Borel equivariant homology is valid for any $G$ and any family $\mathcal{F}$ of subgroups of $G$.

For the remainder of this section, let $\mathfrak{F}$ denote the family of finite subgroups of a group, so that for any $G$ a model for $E(\mathfrak{F}, G)$ is the same thing as a model for $\underline{E} G$. Now suppose that $\mathcal{K}_{*}^{?}$ is a generalized equivariant homology theory in the sense of [21] such that the $\left(\mathcal{K}_{*}^{?}, \mathfrak{F}, H\right)$ assembly conjecture holds whenever $H$ is a CAT(0) cubical group, i.e., whenever $H$ admits a cocompact cubical action on a CAT(0) cubical complex with stabilizers in $\mathfrak{F}$. Suppose further that $\mathcal{K}_{*}^{?}$ is continuous in the sense that for any group $G$, and any expression for $G$ as a directed union $G=\cup_{i} G_{i}$, one has that the natural map

$$
\lim _{i} \mathcal{K}_{*}^{G_{i}}(*) \rightarrow \mathcal{K}_{*}^{G}(*)
$$

is an isomorphism [1.

Theorem 10.1. For a group $G$ and a model $X$ for $\underline{\mathrm{E}} G$, define a group $\widetilde{G}$ and a homomorphism $\alpha: \widetilde{G} \rightarrow G$ as in the statement of Theorem 8.3. For a generalized equivariant homology theory $\mathcal{K}_{*}^{\text {? }}$ satisfying the hypotheses listed in the previous paragraph, the following statements are equivalent:

- The $\left(\mathcal{K}_{*}^{?}, \mathfrak{F}, G\right)$ assembly conjecture holds

- The map $\operatorname{ind}_{\alpha}: \mathcal{K}_{*}^{\widetilde{G}}(*) \rightarrow \mathcal{K}_{*}^{G}(*)$ is an isomorphism

Proof. First we claim that the $\left(\mathcal{K}_{*}^{?}, \mathfrak{F}, \widetilde{G}\right)$ assembly conjecture holds. Let $\left\{X_{i}: i \in \mathcal{I}\right\}$ denote the collection of $G$-subcomplexes of $X$ that contain only finitely many $G$-orbits of simplices and have the property that $X_{i} / G$ is connected. Then $X$ is the directed union of the subspaces $X_{i}$. Applying the construction of Theorem 8.3 to these spaces $X_{i}$ gives an expression for $\widetilde{G}$ as a directed union of subgroups $\widetilde{G}_{i}$. The universal cover of $T_{X_{i}}$ is a CAT $(0)$ cubical complex admitting a cocompact action of $\widetilde{G}_{i}$ with stabilizers in $\mathfrak{F}$. Hence the $\left(\mathcal{K}_{*}^{?}, \mathfrak{F}, \widetilde{G}_{i}\right)$ assembly conjecture holds for each $i \in \mathcal{I}$. Since $\mathcal{K}_{*}^{?}$ is assumed to be continuous, the assembly map for $\widetilde{G}$ is equal to the following composite:

$$
\mathcal{K}_{*}^{\widetilde{G}}(\underline{\mathrm{E}} \widetilde{G}) \cong \lim _{i} \mathcal{K}_{*}^{\widetilde{G}_{i}}\left(\underline{\mathrm{E}} \widetilde{G}_{i}\right) \rightarrow \lim _{i} \mathcal{K}_{*}^{\widetilde{G}_{i}}(*) \cong \mathcal{K}_{*}^{\widetilde{G}}(*) .
$$

It follows that the $\left(\mathcal{K}_{*}^{?}, \mathfrak{F}, \widetilde{G}\right)$ assembly conjecture holds as claimed. 
The homomorphism $\alpha: \widetilde{G} \rightarrow G$ induces an equivariant map $\underline{\mathrm{E}} \widetilde{G} \rightarrow \underline{\mathrm{E}} G$ and hence a commutative square as below:

$$
\begin{array}{cccc}
\mathcal{K}_{*}^{\widetilde{G}}(\underline{\mathrm{E}} \widetilde{G}) & \rightarrow & \mathcal{K}_{*}^{G}(\underline{\mathrm{E} G}) \\
\downarrow & & \downarrow \\
\mathcal{K}_{*}^{\widetilde{G}}(*) & \rightarrow & \mathcal{K}_{*}^{G}(*),
\end{array}
$$

in which the horizontal maps are both called ind $\mathrm{i}_{\alpha}$, and come from the identifications $\operatorname{ind}_{\alpha}(\underline{\mathrm{E}} \widetilde{G})=\underline{\mathrm{E}} G$ and $\operatorname{ind}_{\alpha}(*)=*$. By Theorem 8.3, the map down the left side of the diagram is an isomorphism, and by the argument given in the first paragraph the top horizontal map is an isomorphism. It follows that the lower horizontal map is an isomorphism if and only if the map down the right side is an isomorphism.

There are a number of assembly conjectures to which Theorem 10.1 applies. In each case discussed below, the smoothness of the relevant homology theory is established in $\mathbf{1}$.

- The Bost conjecture is the assembly conjecture for a homology theory in which $\mathcal{K}_{*}^{G}(*)$ is isomorphic to the topological $K$-theory of $\ell^{1}(G)$. In [27] it is shown that CAT(0) cubical groups have the Haagerup property. In [18 the Bost conjecture is established for any group having the Haagerup property. Thus Theorem 10.1 applies to this case.

- Theorem 10.1 does not apply directly to the Baum-Connes conjecture, an assembly conjecture for which $\mathcal{K}_{*}^{G}(*)$ is isomorphic to the topological $K$-theory of the reduced group $C^{*}$-algebra $C_{r}^{*}(G)$. The Baum-Connes conjecture holds for groups having the Haagerup property [14, and the homology theory is smooth [1. The difficulty is that the homomorphism $\alpha: \widetilde{G} \rightarrow G$ does not induce a homomorphism from $C_{r}^{*}(\widetilde{G})$ to $C_{r}^{*}(G)$, and so there is no known way to define the map ind ${ }_{\alpha}$ appearing in the statement of Theorem 10.1 Instead of Theorem 10.1 we can make the following slightly weaker statement: with notation as in the statement of Theorem 10.1 and with $\mathcal{K}_{*}^{?}$ equal to the generalized equivariant homology theory appearing in the Baum-Connes conjecture, we have that the Baum-Connes conjecture holds for $\widetilde{G}$ and $\operatorname{ind}_{\alpha}: \mathcal{K}_{*}^{\widetilde{G}}(\underline{\mathrm{E}} \widetilde{G}) \rightarrow \mathcal{K}_{*}^{G}(\underline{\mathrm{E} G})$ is an isomorphism. In the special case when $G$ is torsion-free (and with a different construction of a group $\widetilde{G}$ ) a similar statement appears in [5, Introduction] and [24, part I, theorem 5.19].

- Let $R$ be a ring equipped with an involution. After inverting the prime 2, the Farrell-Jones conjecture in algebraic $L$-theory with coefficients in $R$ is equivalent to to the $\left(\mathcal{K}_{*}^{?}, \mathfrak{F}, G\right)$ assembly conjecture, where $\mathcal{K}_{*}^{H}(*)$ is isomorphic to $\mathbb{Z}[1 / 2] \otimes \mathbf{L}_{*}^{\langle-\infty\rangle}(R H)$, the algebraic $L$ groups of $R H$ localized away from the prime 2. The Farrell-Jones conjecture in algebraic $L$-theory is proved for CAT(0) groups in [2], and hence Theorem 10.1 applies directly for this choice of $\mathcal{K}_{*}^{\text {? }}$.

- Let $R$ be a regular ring, and suppose that $G$ is a group with the property that the order of every finite subgroup of $G$ is a unit in $R$. Under these circumstances the Farrell-Jones conjecture for the algebraic $K$-theory of $R G$ is equivalent to the $\left(\mathcal{K}_{*}^{?}, \mathfrak{F}, G\right)$ assembly conjecture where $\mathcal{K}_{*}^{?}$ is a generalized equivariant homology theory for which $\mathcal{K}_{*}^{G}(*)$ is isomorphic to the algebraic $K$-theory of $R G$. (Without these extra assumptions on $G$ and $R$, the Farrell-Jones conjecture would require the larger family of virutally cyclic subgroups rather than the family $\mathfrak{F}$ of finite subgroups.) The Farrell-Jones conjecture for CAT(0) groups is proved in $\mathbf{3 2}$ (see also [2]). Every finite subgroup of the group $\widetilde{G}$ in Theorem 10.1 is isomorphic to a subgroup of $G$. Thus provided that $R$ satisfies the hypotheses listed at the start of this paragraph, Theorem 10.1 applies in this case. Thus the Farrell-Jones assembly map $\mathcal{K}_{*}^{G}(\underline{\mathrm{E} G}) \rightarrow \mathcal{K}_{*}^{G}(*)$ is an isomorphism if and only if $\alpha$ induces an isomorphism from $K_{*}(R \widetilde{G})$ to $K_{*}(R G)$. 


\section{The torsion subgroup of a $C A T(0)$ group}

In this section, we let $\mathbf{C}$ denote the class of groups that act cocompactly by cellular isometries on a $\mathrm{CAT}(0)$ cubical complex, and that contain a torsion-free subgroup of index at most two. A simple way to describe a group $G$ in $\mathbf{C}$ is by giving a finite locally $\mathrm{CAT}(0)$ cubical complex $T$ together with a cellular involution $\tau$ on $T$ : the group $G$ thus described is the group of all lifts to the universal cover of $T$ of elements of the group generated by $\tau$, and the fundamental group of $T$ is a torsion-free subgroup of $G$ of index at most two. Note that we allow the case when $\tau$ is the identity map on $T$, in which case $G$ is equal to the fundamental group of $T$.

Note that it is easy to compute the number of conjugacy classes of elements of order two in $G$ : if $\tau$ is not the identity map then there is a bijection between these conjugacy classes and the components of the fixed point set $T^{\tau}$. Not every problem involving the torsion in $G$ can be solved so easily however, as the following theorem shows.

Theorem 11.1. There is no algorithm to determine for all $G \in \mathbf{C}$ whether $G$ is generated by torsion.

Proof. It is known that there is no algorithm to determine whether a given finite presentation presents the trivial group. In particular, we may construct a sequence $X_{n}$ of finite connected simplicial complexes such that no algorithm can compute the function $f: \mathbb{N} \rightarrow\{0,1\}$ defined by $f(n)=1$ if and only if $\pi_{1}\left(X_{n}\right)$ is trivial.

Apply Theorem A to the sequence $X_{n}$, and consider the corresponding sequence $\left(T_{X_{n}}, \tau\right)$ of finite locally CAT(0) cubical complexes equipped with a cellular involution, which describes a sequence $G_{n}$ of groups in $\mathbf{C}$. The group $G_{n}$ is generated by torsion if and only if $\pi_{1}\left(X_{n}\right)$ is trivial, and so there can be no algorithm to decide whether or not each $G_{n}$ is generated by torsion.

\section{Closing remarks}

It is natural to ask whether there is a version of Theorem A in which 'locally CAT(0)' is replaced by the stronger condition 'locally $\operatorname{CAT}(-1)$ '. The author posed this question in 4 , Q 1.23]. As a first step in answering this question which is of interest in its own right: can one construct high-dimensional locally CAT $(-1)$ spaces that are acyclic but not contractible?

In [15, theorem 20.2], Januszkiewicz and Swiattkowski show that for any finite simplicial complex $X$, there exists a CAT $(-1)$ simplicial complex $S_{X}$ and a group $H_{X}$ acting properly cocompactly and simplicially on $S_{X}$, such that the quotient $S_{X} / H_{X}$ is homotopy equivalent to $X$. This result can be viewed as a $\operatorname{CAT}(-1)$ but non-natural version of property (ii) of Theorem A for finite complexes. Corollaries 20.4 and 20.5 of $\mathbf{1 5}$ answer a question posed by the author in [4, Q 1.24].

Two questions that were raised in earlier versions of this paper have been answered. Polák and Wise have shown that the group presented in Proposition 3.2 is residually finite [28. Raeyong Kim has shown that every finite connected $X$ has the same homology as some locally $\operatorname{CAT}(0)$ cubical complex $V_{X}$ whose fundamental group is a duality group [17; this is a $\operatorname{CAT}(0)$ version of a theorem of Hausmann $\mathbf{1 3}$.

Finally, a comment on hyperbolization as in [11, sec. 3.4] and 9. As in our Kan-Thurston construction, one constructs for a simplicial complex $X$, a locally CAT(0) cubical complex $W_{X}$ equipped with a map $w_{X}: W_{X} \rightarrow X$. The extra property enjoyed by $W_{X}$ is that its vertex links are similar to those in $X$, so that if $X$ is a PL $n$-manifold then so is $W_{X}$. The map on homology induced by $w_{X}$ can be taken to be a split surjection. Consideration of the case when 
$X$ is a 2-sphere shows that there cannot be a 'Kan-Thurston hyperbolization' in general. For $n>1$ it seems to be unknown whether there exists a aspherical homology $2 n$-sphere.

\section{Appendix A. Metrizing complexes}

We collect some facts about simplicial and cubical complexes, together with detailed references or proofs. Since we intend to metrize our complexes, we refer to [8, I.7.40], parts (2) and (3) respectively for the definitions of a simplicial complex and a cubical complex.

Throughout this section, each $n$-cube is metrized as a standard Euclidean cube of side length 1 , and each $n$-simplex is metrized as the subspace of the Riemannian sphere of radius 1 in $\mathbb{R}^{n+1}$ defined by intersecting the sphere with $\left(\mathbb{R}_{\geq 0}\right)^{n+1}$. This metric on the $n$-simplex will be called the 'all right metric'. Let $C$ denote either a simplicial complex or a cubical complex, and refer to the simplices or cubes of $C$ as its cells. As in [8, I.5.9], the metric on the cells induces a pseudo-metric on any connected simplicial or cubical complex, in which the distance between points $x$ and $y$ is the infimum of sums of the form $\sum_{i=1}^{n} d\left(x_{i-1}, x_{i}\right)$, where $x_{0}=x, x_{n}=y$, and for each $i$, there is a single cell containing $x_{i}$ and $x_{i-1}$, and the distance is measured in the standard metric on that cell. (A sequence of points $x_{0}, \ldots, x_{n}$ with these properties is called a 'string' or an ' $n$-string'.) By [8, I.7.10], this pseudo-metric turns out to be a metric. Moreover, this metric makes $C$ into a length space as defined in [8, I.3.1]. Another treatment of this material is contained in [25, section 3]. In the case when $C$ is finite-dimensional, it is shown in [8, I.7.13] that this metric is complete. We shall characterize the complexes $C$ for which the metric is complete, but first we need some lemmas.

Proposition A.1. Let $\sigma$ be a cell of $C$ and $x$ a point of $C$, such that $d=d(\sigma, x)$ satisfies either $d<1$ if $C$ is cubical or $d<\pi / 2$ if $C$ is simplicial. Then there is straight line segment from $\sigma$ to $x$ of length $d$, which is contained in the smallest closed cell of $C$ containing $x$. This line segment is unique.

Proof. Pick an $n$ and an $n$-string $x_{0}, \ldots, x_{n}$ of length less than 1 (resp. $\pi / 2$ ) with $x_{0} \in \sigma$ and $x_{n}=x$, and suppose that $n \geq 2$. Let $\sigma_{i}$ be the minimal closed cell containing the line segment $\left[x_{i-1}, x_{i}\right]$. We may assume that no $\sigma_{i}$ is a face of $\sigma$. If the dimensions of these cells satisfy $\operatorname{dim}\left(\sigma_{2}\right)>\operatorname{dim}\left(\sigma_{1}\right)$, then $\sigma_{1}$ is a face of $\sigma_{2}$ and the $(n-1)$-string $x_{0}, x_{2}, \ldots, x_{n}$ is shorter. If $\sigma_{1}=\sigma_{2}$, then once again the $(n-1)$-string $x_{0}, x_{2}, \ldots, x_{n}$ is either shorter or of the same length (the latter possibility occurs if $x_{1}$ is on the line segment $\left[x_{0}, x_{2}\right]$ ). In the remaining case, the minimal closed cell $\tau$ containing $x_{1}$ is a proper face of $\sigma_{1}$. Since the segment $\left[x_{0}, x_{1}\right]$ has length less than 1 (resp. $\pi / 2$ ), it follows that $\tau \cap \sigma$ is non-empty. Let $x_{0}^{\prime}$ be the base of the perpendicular from $x_{1}$ to $\tau \cap \sigma$. The $n$-string $x_{0}^{\prime}, x_{1}, \ldots, x_{n}$ is a path from $\sigma$ to $x$ and is shorter than the original string.

This process one can reduce any $n$-string for $n \geq 2$ to the single line segment $[y, x]$, where $\tau$ is the minimal closed cell containing $x$ and $y$ is the base of the perpendicular in $\tau$ from $x$ to $\tau \cap \sigma$. It follows that this segment is the unique shortest path from $\sigma$ to $x$, and that it has length $d$.

Corollary A.2. Suppose that $\lambda<1$ in the cubical case or $\lambda<\pi / 2$ in the simplicial case, and for $\sigma$ a cell of $C$, let $O=O_{C}^{\lambda}(\sigma)$ be the open $\lambda$-ball in $C$ around $\sigma$. For any cell $\tau$ in $C$, one has that

$$
O_{C}^{\lambda}(\sigma) \cap \tau=O_{\tau}^{\lambda}(\sigma \cap \tau) .
$$

In particular $O_{C}^{\lambda}(\sigma) \cap \tau$ is empty if $\sigma \cap \tau$ is empty. 
Corollary A.3. Let $\lambda=2 / 3$ and for $\tau$ a cube in a cubical complex $C$ define $F_{\tau}$ by the equation

$$
F_{\tau}=C-\bigcup_{\sigma \cap \tau=\emptyset} O_{C}^{\lambda}(\sigma) .
$$

Also define $F_{\emptyset}=\emptyset$. Then $F_{\tau}$ is closed and contains the closed ball in $C$ of radius $1 / 3$ about $\tau$. If $\tau_{i}$ for $i \in I$ are cells of $C$, with intersection $\mu$, then $\bigcap_{i \in I} F_{\tau_{i}}=F_{\mu}$.

Proof. As the complement of a union of open sets, $F_{\tau}$ is closed. The previous corollary allows one to find the intersection of $F_{\tau}$ with each closed cell of $C$. This gives a combinatorial description of $F_{\tau}$. Let $C^{\prime}$ be the subdivision of $C$ in which each $n$-cube is divided in to $3^{n}$ congruent pieces. Then $F_{\tau}$ is equal to the union of the (closed) cubes in $C^{\prime}$ that intersect $\tau$. Equivalently, $F_{\tau}$ is equal to the union of the (closed) cubes in $C^{\prime}$ that contain the barycentre of some cube of $C$. The other claimed properties follow.

Proposition A.4. Suppose that there is a point $x$ in a cubical complex $C$ which is at distance at most $1 / 3$ from a collection $\tau_{i}$ for $i \in I$ of cells of $C$. Then $\mu=\bigcap_{i \in I} \tau_{i}$ is non-empty.

Proof. The point $x$ is in each $F_{\tau_{i}}$, and hence $\bigcap_{i \in I} F_{\tau_{i}}=F_{\mu}$ is non-empty.

REMARK A.5. There is no analogue of this proposition for all right simplicial complexes. Let $C$ be the boundary of an $n$-simplex. The top dimensional faces of $C$ do not intersect, but their barycentres are at distance $\arccos (1-1 / n)$ from each other.

Theorem A.6. The metric on $C$ is complete if and only if $C$ does not contain an infinite ascending sequence of cells.

Proof. Let $\sigma_{0} \subseteq \sigma_{1} \subseteq \cdots$ be an ascending sequence of cells, where $\sigma_{i}$ has dimension $i$. Let $x_{0}=\sigma_{0}$, and for $i>0$ pick $x_{i} \in \sigma_{i}$ to be a point at distance $1 / 3^{i}$ from the boundary of $\sigma_{i}$ whose nearest point in the boundary is $x_{i-1}$. This is a Cauchy sequence with no convergent subsequence.

For the converse, first consider the case when $C$ is cubical. Suppose that $C$ contains no infinite ascending sequence of cells. Let $\left(x_{n}\right)$ be a Cauchy sequence in $C$, and let $\sigma_{n}$ denote the minimal closed cube containing $x_{n}$. By passing to a subsequence, we may assume that each $d\left(x_{m}, x_{n}\right)<1 / 3$, and so by Proposition A.4, $\theta=\bigcap_{n} \sigma_{n}$ is non-empty. Suppose that $\theta^{\prime}$ is a cell properly containing $\theta$ which is contained in $\sigma_{n}$ for infinitely many $n$. By passing to this subsequence, we may replace $\theta$ by $\theta^{\prime}$. Since there is no infinite ascending chain of cells, we may assume that $\theta$ has the property that for each $\theta^{\prime}>\theta$, there are only finitely many $n$ for which $\theta^{\prime} \geq \sigma_{n}$. Now let $y_{n}$ be the closest point of $\theta$ to $x_{n}$. The map $x_{n} \mapsto y_{n}$ is distance-decreasing, so $\left(y_{n}\right)$ is Cauchy. (The reader who does not want to check that this map is distance-decreasing may instead pass to a convergent subsequence.) Let $y$ be the limit of the sequence $\left(y_{n}\right)$.

It remains to show that $\left(x_{n}\right)$ converges to $y$. But if $d\left(x_{m}, x_{n}\right)<1$, then certainly $d\left(\sigma_{m}, x_{n}\right)<$ 1, and so by Proposition A.1, the shortest path from $x_{n}$ to $\sigma_{m}$ is the straight line inside $\sigma_{n}$ from $x_{n}$ to $y$. Thus $d\left(y, x_{n}\right)=d\left(\sigma_{m}, x_{n}\right) \leq d\left(x_{m}, x_{n}\right)$. This completes the proof in the cubical case.

For the simplicial case, given an all right simplicial complex $C$ with vertex set $V$, let $v_{0}$ be the origin in $W=\bigoplus_{V} \mathbb{R}$, equipped with the standard Euclidean norm, and identify the vertex $v \in C$ with the unit vector in the positive $v$-direction in $W$. For each $(n-1)$-simplex 
$\sigma=\left(v_{1}, \ldots, v_{n}\right)$ of $C$, let $c(\sigma)$ be the $n$-cube in $W$ with vertex set the points $\sum_{i=1}^{n} \epsilon_{i} v_{i}$, for $\epsilon_{i} \in\{0,1\}$. Define a cubical complex $D$ as the union of all of these (closed) cubes. Now $D$ is a cubical complex in which the link of $v_{0}$ is equal to $C$. In particular, $D$ has no infinite ascending sequence of cubes if and only if $C$ has no infinite ascending sequence of simplices. Futhermore, there is a natural embedding of $C$ in $D$, which embeds $\sigma$ as the intersection of $c(\sigma)$ and the unit sphere in $W$ based at $v_{0}$. This embedding does not preserve distances: if $x, y$ are points inside a single simplex $\sigma$ of $C$ and $d_{\sigma}(x, y)=\theta$, then $d_{c(\sigma)}(x, y)=2 \sin (\theta / 2)$. The convexity properties of the sine function imply that for any points $x, y \in C$ with $d_{C}(x, y)=\theta \leq \pi / 4$, one has that $2 \sin \left(d_{C}(x, y) / 2\right) \leq d_{D}(x, y) \leq d_{C}(x, y)$. It follows that a sequence $\left(x_{n}\right)$ in $C$ is Cauchy in $C$ if and only if it is Cauchy in $D$. If $x \in D$ is the limit in $D$ of the Cauchy sequence $\left(x_{n}\right)$ in $C$, then by continuity of the metric, $d_{D}\left(v_{0}, x\right)=1$ and so $x \in C$.

Theorem A.7. The identity map from $C$ with the $C W$-topology to $C$ with the metric topology is a homotopy equivalence.

Proof. This is due to Dowker [10]. In the cubical case, there is an easy combinatorial description of a homotopy inverse to the identity map. Let $C^{\prime}$ denote the subdivision of $C$ in which each $n$-cube is cut into $3^{n}$ cubes. There is a function $f$ from the set of cubes of $C^{\prime}$ to the set of cubes of $C$, defined by $f(\sigma)=\tau$ if the closed cube $\sigma$ contains the barycentre of $\tau$. For each $\tau \in C$, the set $f^{-1}(\tau)$ contains a unique cube $m(\tau)$ of minimal dimension. The cube $m(\tau)$ is the 'middle cube' of $\tau$, and its dimension is equal to the dimension of $\tau$. Every cube $\sigma \in f^{-1}(\tau)$ has $m(\tau)$ as a face. Define a map $\phi: C^{\prime} \rightarrow C$ as follows. If $\sigma=m(\tau)$ for some $\tau$, then define $\phi: \sigma \rightarrow \tau$ by expanding all distances from the common barycentre of $\sigma$ and $\tau$ by a factor of 3. Otherwise, take $\phi$ to be the composition of the orthogonal projection from $\sigma$ to $m(f(\sigma))$ with the map already defined from $m(f(\sigma))$ to $f(\sigma)$.

This map $\phi$ is cellular as a map from $C$ to $C$, and multiplies distances by a factor of at most 3. It follows that in either topology there is a homotopy from $\phi$ to the identity map which is linear on each cube. It remains to check that $\phi$ is continuous from the metric topology to the CW-topology. To see this, let $x$ be a point of $C$, and let $\sigma$ be the minimal cube of $C$ that contains $\phi(x)$. If $O$ is any open set in $C$ containing $\phi(x)$, pick $\epsilon>0$ such that the intersection of the interior of $\sigma$ and $O$ contains the open $\epsilon$-ball around $\phi(x)$. Then $\phi^{-1}(O)$ contains the open $\epsilon / 3$-ball in $C$ around $x$. This shows that $\phi$ is continuous.

Remark A.8. The set $F_{\tau}$ defined in Corollary A.3 is equal to $\phi^{-1}(\tau)$. This fact can be used to give a different proof of Corollary A.3.

Appendix B. The CAT $(\kappa)$ conditions

Definition B.1. Define a combinatorially CAT(0) cubical complex to be a simply connected cubical complex in which all vertex links are flag. (It will be shown in Theorem B.8 that a cubical complex is $\operatorname{CAT}(0)$ if and only if it is combinatorially $\operatorname{CAT}(0)$.)

Definition B.2. A full subcomplex of a simplicial complex $C$ is a subcomplex $D$ such that whenever $\sigma \in C$ and each vertex $v$ of $\sigma$ is in $D$, then $\sigma$ is in $D$. A combinatorially convex subcomplex of a cubical complex $C$ is a connected subcomplex $D$ such that for each vertex $v$ of $D$, the link in $D, \operatorname{Lk}_{D}(v)$ is a full subcomplex of $\operatorname{Lk}_{C}(v)$. 
Proposition B.3. Every finite subcomplex of a simplicial complex $C$ is contained in some finite full subcomplex $D$. If $C$ is connected, then $D$ may be taken to be connected.

Proof. Let $C^{\prime}$ be a finite subcomplex of $C$, and let $D$ be the full subcomplex of $C$ with the same vertex set as $C^{\prime}$. This is a finite full subcomplex since its vertex set is finite. If $C^{\prime}$ is connected, then so is $D$. In the case when $C$ is connected, any finite subcomplex $C^{\prime \prime}$ is contained in a finite connected subcomplex $C^{\prime}$ (e.g., the union of $C^{\prime \prime}$ and a choice of edge paths joining the components of $C^{\prime \prime}$ ), and so the extra claim in this case follows.

Theorem B.4. Every finite subcomplex of a combinatorially CAT(0) cubical complex is contained in a finite combinatorially convex subcomplex.

Proof. This uses Sageev's theory of hyperplanes [31. A hyperplane in a combinatorially CAT(0) cubical complex $C$ is an equivalence class of directed edges under the transitive closure of the relation 'there exists a square in which the two directed edges are parallel'. The corresponding open hyperplane neighbourhood is the union of all open cubes whose closures contain an edge of the hyperplane. If $H$ is an open hyperplane neighbourhood, then $C-H$ is a cubical complex with the same vertex set as $C$. If $v$ is a vertex of $C$, then $\operatorname{Lk}_{C-H}(v)$ is the full subcomplex of $\operatorname{Lk}_{C}(v)$ with vertex set the (undirected) edges containing $v$ that are not contained in $H$. We define the opposite hyperplane to $H$ to be the hyperplane which contains the reverse of every oriented edge in $H$. We quote four results which can easily be deduced from Sageev's paper [31. For any square $\sigma \in C$, a hyperplane contains at most two of the eight directed edges of $\sigma$ [31, lemma 4.8 and corollary 4.9]. For each open hyperplane neighbourhood $H, C-H$ has two components [31, theorem 4.10] (this implies that no hyperplane is equal to its own opposite). Call these two components the half-spaces defined by $H$. If the shortest 1-skeleton path between vertices $v$ and $w$ has length $n$, then there are exactly $n$ opposite pairs of hyperplanes in $C$ that separate $v$ and $w$ (the lower bound comes from [31, theorem 4.13], and the upper bound from [31, theorem 4.6]). A vertex $v$ of $C$ is uniquely determined by the collection of half-spaces in which it lies (follows from [31, theorems 4.6 and 4.10], since if $e$ is an edge on any 1-skeleton geodesic from $v$ to $w$ then the hyperplane containing $e$ separates $v$ and $w)$.

Given a finite subcomplex $C^{\prime}$ of $C$, let $V^{\prime}$ be the vertex set of $C^{\prime}$, and let $D$ be the intersection of all half-spaces of $C$ that contain $V^{\prime}$. As an intersection of combinatorially convex subcomplexes, $D$ is combinatorially convex. Suppose that $V^{\prime}$ contains $n$ elements and that each pair $v, w \in C$ can be joined by a 1-skeleton path of length at most $N$ in $C$. Then there are at most $M=N\left(\begin{array}{l}n \\ 2\end{array}\right)$ hyperplanes of $C$ that separate points of $V^{\prime}$. Since vertices of $C$ are uniquely determined by the half-spaces that contain them, it follows that $D$ contains at most $2^{M}$ vertices.

Before proving the main theorems of this section, we recall a definition from [8, I.7.8]. Suppose that $C$ is a simplicial or cubical complex in which cells have been given compatible metrics (i.e., so that the inclusion of each face of each cell is an isometry). Under these circumstances, for $x$ a point of $C$ and $\sigma$ any cell of $C$ containing $x$, define $\epsilon(x, \sigma)$ to be the infimum of the distances from $x$ to points in closed faces of $C$ that do not contain $x$. Now define $\epsilon_{C}(x)$ to be the infimum of the numbers $\epsilon(x, \sigma)$ as $\sigma$ ranges over all closed cells of $C$ that contain $x$. Informally, we may think of $\epsilon(x)$ as the infimum of the distances from $x$ to points $y$ in closed cells that do not contain $x$. 
Proposition B.5. Let $C$ be either an all-right simplicial complex or a cubical complex. Then for any point $x$ of $C$, we have that $\epsilon_{C}(x)=\epsilon_{\sigma}(x)$, where $\sigma$ is the unique closed cell of $C$ such that $x$ is in the interior of $\sigma$. In particular, $\epsilon_{C}(x)>0$ for all $x$.

Proof. An easy exercise.

Theorem B.6. Let $C$ be an all-right simplicial complex. Then $C$ is $C A T(1)$ if and only if $C$ is flag.

Proof. The case when $C$ is finite dimensional is proved in [8, II.5.18]. The argument given there to show that CAT(1) implies flag generalizes easily to the infinite-dimensional case. If $C$ is not flag, take a 'missing simplex' of minimal dimension, i.e., a subset $v_{0}, \ldots, v_{n}$ of the vertex set of $C$, where $n$ is at least 2 and is minimal subject to the property that the set $\left\{v_{0} \ldots, v_{n}\right\}$ does not span a simplex of $C$ but any proper subset does. If $n=2$, then the union of the three edges joining the vertices $v_{0}, v_{1}, v_{2}$ is a closed local geodesic of length less than $2 \pi$, showing that $C$ cannot be CAT(1). For $n>2$, argue by induction on $n$. The link $\operatorname{Lk}_{C}\left(v_{n}\right)$ contains the 'missing simplex' $v_{0}, \ldots, v_{n-1}$ of dimension $n-1$, and so $\operatorname{Lk}_{C}\left(v_{n}\right)$ is not CAT(1). It follows from [8, II.5.2] that $C$ cannot be CAT(1). (In the infinite dimensional case, the hypotheses of [8, II.5.2] are not satisfied, but Proposition B.5 shows that the alternative hypotheses listed in [8, II.5.3] do hold.)

The general case of 'flag implies CAT(1)' will be proved below.

Theorem B.7. Let $i: D \rightarrow C$ be the inclusion of a full subcomplex in a flag simplicial complex. Then $i$ preserves distances less than $\pi$, in the sense that for $x, y \in D$, one has that $d_{D}(x, y)<\pi$ if and only if $d_{C}(i(x), i(y))<\pi$ and if this holds, then $d_{D}(x, y)=d_{C}(i(x), i(y))$.

Proof. The proof will rely on Theorem B.6, so for now it will only cover the case when $C$ is finite-dimensional. This case will then be used to prove the general case of Theorem B.6, and then the proof given below will cover all cases.

Write $C *_{D} C$ for the double of $C$ along $D$, i.e., the quotient space obtained from two copies of $C$ by identifying the two copies of $D$. Each of the two inclusions $C \rightarrow C *_{D} C$ is a map that does not increase distance (since a string in $C$ maps to a string in $C *_{D} C$ of the same length). The composite map

$$
C \rightarrow C *_{D} C \rightarrow C *_{C} C=C
$$

is the identity, and hence each of the inclusions $C \rightarrow C *_{D} C$ is an isometric embedding.

It is easily seen that $C *_{D} C$ is flag. By the cases of Theorem B.6 that are already known, if $C$ is finite-dimensional, then $C, D$ and $C *_{D} C$ are all CAT(1). The map $i: D \rightarrow C$ does not increase distance. The self-map of $C *_{D} C$ that swaps the two copies of $C$ is an isomorphism and so is an isometry. Suppose that $x, y \in D$ are such that $d_{C}(i(x), i(y))<\pi$. Then by 8 , II.1.4(1)], there is a unique geodesic in $C *_{D} C$ from $x$ to $y$. If the geodesic did not lie entirely inside $D$, there would be at least two such geodesics. Hence the geodesic does lie inside $D$, and so $d_{D}(x, y)=d_{C}(x, y)$.

Proof. (Theorem B.6, general case.) Let $C$ be a flag complex. Given any two points $x, y \in C$ at distance less than $\pi$, take a string $\gamma=x_{0}, \ldots, x_{m}$ in $C$ from $x$ to $y$ whose length is less than $\pi$. Now let $D$ be a finite full subcomplex of $C$ that contains the path defined by the string $\gamma$. Then $d_{D}(x, y)<\pi$ since $D$ contains the string $\gamma$. Hence there is a geodesic string $\gamma^{\prime}=y_{0}, \ldots, y_{n}$ 
from $x$ to $y$ in $D$. Claim that the string $\gamma^{\prime}$ is also a geodesic in $C$. To see this suppose not. In that case $C$ would contain a shorter string $\gamma^{\prime \prime}$. Now let $D^{\prime}$ be a finite full subcomplex of $C$ containing both $D$ and the path defined by $\gamma^{\prime \prime}$. By Theorem B.7, the inclusion of $D$ into $D^{\prime}$ preserves distances less than $\pi$. This contradiction shows that $\gamma^{\prime}$ is a geodesic in $C$, and so any two points at distance less than $\pi$ are joined by a geodesic.

Now suppose one is given a geodesic triangle in $C$ of perimeter less than $2 \pi$, and two points $x$ and $y$ on the perimeter of this triangle. Then $x$ and $y$ are separated by less than $\pi$ so there exists a geodesic between them. Now the three sides of the triangle together with the geodesic from $x$ to $y$ are contained in a finite full subcomplex $D$ of $C$. Since $D$ is flag, the distance from $x$ to $y$ in $D$ is no greater than the distance between corresponding points on the perimeter of a triangle with the same side lengths in the standard sphere $S^{2}$. But by the argument given above, $d_{D}(x, y)=d_{C}(x, y)$ and so the CAT(1) inequality holds.

Proof. (Theorem B.7, general case.) The proof given above in the finite-dimensional case is valid in general now that the general case of Theorem B.6 is known.

Theorem B.8. (Gromov) A simply connected cubical complex is CAT(0) if and only if its vertex links are flag complexes.

Proof. The finite-dimensional case is [8, II.5.20]. If $C$ is CAT(0), then by [8, II.5.2], for each vertex $v, \operatorname{Lk}_{C}(v)$ is CAT(1). (Just as in the proof of Theorem B.6, the hypotheses of [8, II.5.2] do not hold in the infinite-dimensional case, but Proposition B.5 ensures that the alternative hypotheses listed in [8, II.5.3] hold.) Hence by Theorem B.6, each vertex link is a flag complex.

The proof of the general case of 'flag links implies CAT(0)' will be given below.

Theorem B.9. Let $C$ be a simply-connected cubical complex with all vertex links flag, and let $D$ be a combinatorially convex subcomplex of $D$. Then the map $i: D \rightarrow C$ is isometric.

Proof. The relationship between the proofs of Theorem B.8 and Theorem B.9 is similar to the relationship between the proofs of Theorem B.6 and Theorem B.7. More precisely, this proof will rely on Theorem B.8, and so for now it will only cover the case when $C$ is finitedimensional. As before, consider the double of $C$ along $D, C *_{D} C$. The argument given in the proof of Theorem B.7 shows that each of the two inclusions of $C$ into $C *_{D} C$ is an isometric embedding, and there is an isometric involution of $C *_{D} C$ swapping the two copies of $C$ whose fixed point set is $D$. By van Kampen's theorem, $C *_{D} C$ is simply-connected. The link of each vertex of $C *_{D} C$ is flag. From the cases of Theorem B.8 that are already proved, it follows that when $C$ is finite-dimensional, $C *_{D} C$ is $\operatorname{CAT}(0)$. Given any two points $x, y \in D$, there is a unique geodesic between them in $C *_{D} C$. By uniqueness, this geodesic must lie inside $D$, and hence $d_{D}(x, y)=d_{C}(x, y)$.

Lemma B.10. Any finite combinatorially convex subcomplex of a combinatorially CAT $(0)$ cubical complex is contractible.

Proof. Let $D$ be a finite combinatorially convex subcomplex of $C$. Since $D$ is finite, the fundamental group of $D$ is finitely generated, and so by attaching finitely many discs to $D$ inside $C$ we may kill the fundamental group of $D$. Hence there exists a finite subcomplex $D^{\prime}$ of $C$ such that the inclusion $D \rightarrow D^{\prime}$ induces the trivial map on fundamental groups. By Theorem B.4 $D^{\prime}$ is contained in a finite combinatorially convex subcomplex $E$ of $C$. Let $\widetilde{E}$ be 
the universal covering of $E$. Since the map $\pi_{1}(D) \rightarrow \pi_{1}(E)$ is trivial, there is a subcomplex of $\widetilde{E}$ which is isomorphic to $D$. Since $\widetilde{E}$ is finite-dimensional, the cases of Theorem B.8 that are already known imply that $\widetilde{E}$ is $\operatorname{CAT}(0)$. By the cases of Theorem B.9 that are already known, the map $i: D \rightarrow \widetilde{E}$ is an isometric embedding. Now for any point $x$ in a $\operatorname{CAT}(0)$ space $X$, there is a contraction from $X$ to $\{x\}$ given by the homotopy $h_{X, x}: X \times I \rightarrow X$ that sends $(y, t)$ to the point at distance $t . d(x, y)$ along the geodesic from $x$ to $y$. If we pick $x$ to be a point of $D$, then the homotopy $h_{\widetilde{E}, x}$ restricts to $D$ as a contraction of $D$ to $x$.

Proof. (Theorem B.8, general case.) Suppose that $C$ is a simply-connected cubical complex in which all links are flag, and let $x$ and $y$ be points of $C$. By Theorem B.4 there is a finite combinatorially convex subcomplex $D$ of $C$ containing both $x$ and $y$. The cases of Theorem B.8 that are already proved imply that $D$ is $\operatorname{CAT}(0)$, since $D$ is simply connected (by Theorem B.9) and vertex links in $D$ are all flag. Thus there is a unique geodesic in $D$ from $x$ to $y$. To see that this path is also a geodesic in $C$, suppose not. Then (as in the proof of Theorem B.6), there is a string $\gamma$ in $C$ of length shorter than $d_{D}(x, y)$. Now let $D^{\prime}$ be a finite combinatorially convex subcomplex of $C$ that contains $D$ and the image of $\gamma$. Then the inclusion of $D$ into $D^{\prime}$ would not be an isometry, contradicting Theorem B.9. This contradiction proves that the geodesic from $x$ to $y$ in $D$ is a geodesic in $C$, and hence $C$ is a geodesic metric space.

Now suppose given a geodesic triangle in $C$ and two points $x$ and $y$ on the perimeter of the triangle. Let $D$ be a finite combinatorially convex subcomplex of $C$ containing the triangle and the geodesic in $C$ from $x$ to $y$. Since all links in $D$ are flag and by Lemma B.10, $D$ is simply connected, it follows that $D$ is $\operatorname{CAT}(0)$. Hence the distance in $D, d_{D}(x, y)$ is no greater than the corresponding distance in a Euclidean comparison triangle. But by choice of $D, d_{D}(x, y)=d_{C}(x, y)$ and so the $\operatorname{CAT}(0)$ inequality holds in $C$.

Proof. (Theorem B.9, general case.) The proof given above in the finite-dimensional case is valid in general now that the general case of Theorem B.8 has been proved.

Theorem B.11. Let $G$ be a finite group acting by isometries on a complete $C A T(0)$ space $X$, or by automorphisms of an arbitrary $C A T(0)$ cubical complex $C$. Then the fixed point set for $G$ is contractible.

Proof. If $x$ and $y$ are fixed points, then the unique geodesic from $x$ to $y$ consists of fixed points. Thus if $x$ is a fixed point, the geodesic contraction of $X$ or $C$ to the point $x$ restricts to a contraction of the fixed point set.

It remains to prove that the fixed point set is non-empty. In the case when $X$ is complete this is proved in [8, corollary II.2.8]. A different proof is given in [6, proposition 3].

If $C$ is a cubical CAT(0) complex, pick a vertex $v$, and construct a finite combinatorially convex subcomplex $D$ containing the orbit $G . v$ as in the proof of Theorem B.4. By the construction, $G$ acts on $D$, and by Lemma B.10 and Theorem B.8 $D$ is CAT(0). Since $D$ is complete, there is a fixed point in $D$.

\section{Appendix C. Cubical and cube complexes}

We work throughout with cubical complexes, rather than the more general complexes referred to as either cube complexes or cubed complexes (see [8, example I.7.40 (4)] for the definition of a cube complex). The main purpose of this section is to show that every $\mathrm{CAT}(0)$ cube complex is in fact cubical, and that the second cubical subdivision of any locally $\mathrm{CAT}(0)$ cube complex is cubical. 

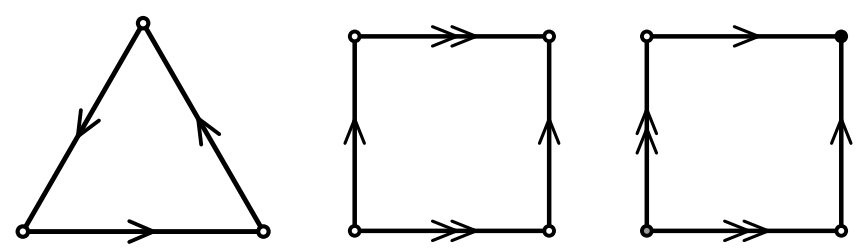

FiguRE C.1. From left to right: a simplex complex which is not a $\Delta$-complex; the torus as a locally $C A T(0)$ cube complex; the sphere as a cube complex.

One extra complication that arises in the general case is that vertex links in cube complexes are not in general simplicial complexes, but are instead a more general type of complex made from simplices. By analogy with the case of cubes, we call these complexes simplex complexes. A simplex complex is an $M_{1}$-polyhedral complex in the sense of [8 definition I.7.37] in which each cell is an all right simplex. Simplex complexes are rather more general than $\Delta$-complexes, which we use in Theorem 5.1, because they lack the ordering on their faces that is implied by a $\Delta$-complex structure.

ExAmples C.1. There is just one $\Delta$-complex with one 0-cell, one 1-cell and one 2-cell, the so-called 'dunces hat', which is contractible. There is another simplex complex with these same numbers of cells, which can be obtained by identifying the three sides of a triangle as shown in Figure C.1.

For an example of a locally CAT(0) cube complex that is not a cubical complex, consider the 2-torus, made by identifying the opposite sides of a square, as shown in Figure C.1.

For an example of a cube complex in which the vertex links are not simplicial complexes, consider the 2-sphere made by identifying the sides of a square as shown in Figure C.1

Lemma C.2. Let $C$ be a cube complex, let $\gamma:[0, d] \rightarrow I^{n}$ be a straight line path joining two points of $I^{n}$, and let $\sigma: I^{n} \rightarrow C$ be a cube of $C$. If the endpoints of $\sigma \gamma$ are distinct points of $C$, then the image of $\sigma \gamma$ is a local geodesic in $C$.

Proof. After possibly replacing $\sigma$ by one of its proper faces, we may assume that the interior of the line segment $\sigma \gamma$ is contained in the interior of the cube $\sigma$. Define $Y_{0}$, a subcomplex of $I^{n}$, to be the union of all faces of $I^{n}$ that do not meet the image of $\gamma$. Let $x^{\prime}=\gamma(0)$ and $y^{\prime}=\gamma(d)$ be the endpoints of $\gamma$, and let $x=\sigma\left(x^{\prime}\right), y=\sigma\left(y^{\prime}\right)$ be the endpoints of $\sigma \gamma$. Define $Y$, a closed subset of $I^{n}$ to be

$$
Y=Y_{0} \cup\left(\sigma^{-1}(x) \backslash\left\{x^{\prime}\right\}\right) \cup\left(\sigma^{-1}(y) \backslash\left\{y^{\prime}\right\}\right) .
$$

Choose $\epsilon>0$ so that the distance in $I^{n}$ between each point of $Y$ and each point of the image of $\gamma$ is strictly greater than $2 \epsilon$. We shall show that each subpath of $\sigma \gamma$ of length at most $\epsilon$ is a geodesic in $C$.

Take $w, z$ points of $\sigma \gamma$ separated by an arc of $\sigma \gamma$ of length $\delta \leq \epsilon$, and suppose that $w=$ $w_{0}, w_{1}, \ldots, w_{m}=z$ is a chain from $w$ to $z$. By the choice of $\epsilon$, each $w_{i}$ must either lie in $\sigma$ or in a higher-dimensional cube of $C$ having $\sigma$ as a face. If any of the $w_{i}$ lie outside $\sigma$, we may obtain a chain of shorter length by replacing each $w_{i}$ by $z_{i}$, defined to be the orthogonal projection of $w_{i}$ into $\sigma$. Any chain in $\sigma$ joining $w$ and $z$ has length greater than or equal to the arc of $\sigma \gamma$ from $w$ to $z$, and the claim follows. 
Remark C.3. Consider the case when $C$ is a Möbius strip made by identifying two opposite sides of a square, $\sigma: I^{2} \rightarrow C$ is the natural identification map, and $\gamma$ is the straight line joining two antipodal vertices of the square. The two endpoints of $\gamma$ are mapped to the same point of $C$, and the image of $\sigma \gamma$ is not a local geodesic in $C$.

Theorem C.4. Let $C$ be any $C A T(0)$ cube complex. Then $C$ is cubical.

Proof. First we show that each cube of $C$ embeds in $C$ and then that the intersection of any two cubes of $C$ is a cube of $C$. Let $\sigma: I^{n} \rightarrow C$ be a cube of $C$, and suppose that $x^{\prime}, y^{\prime}$ are points in the boundary of $I^{n}$ such that $\sigma\left(x^{\prime}\right)=\sigma\left(y^{\prime}\right)$. Let $z^{\prime}$ be the barycentre of $I^{n}$, and let $\gamma, \gamma^{\prime}$ be the straight line paths in $I^{n}$ from $z^{\prime}$ to $x^{\prime}$ and $y^{\prime}$. By Lemma C.2 there exists $\epsilon>0$ so that any subpath of either $\sigma \gamma$ or $\sigma \gamma^{\prime}$ of length at most $\epsilon$ is a geodesic. Since $C$ is $\operatorname{CAT}(0)$, any local geodesic in $C$ is a geodesic [8, II.1.4 (2)], and so $\sigma \gamma$ and $\sigma \gamma^{\prime}$ are geodesics. Since $\sigma\left(x^{\prime}\right)=\sigma\left(y^{\prime}\right)$, these two geodesics have the same endpoints. Since geodesics in a CAT(0) space are unique, it follows that $x^{\prime}=y^{\prime}$ and so $\sigma$ is injective.

Next suppose that $\sigma, \sigma^{\prime}$ are distinct cubes of $C$, and that $a, b \in C$ are points in the intersection of the images of $\sigma$ and $\sigma^{\prime}$. Since $\sigma: I^{m} \rightarrow C$ and $\sigma^{\prime}: I^{n} \rightarrow C$ are injective, there are unique points $x, y \in \partial I^{m}$ and $x^{\prime}, y^{\prime} \in \partial I^{n}$ such that $\sigma(x)=a=\sigma^{\prime}\left(x^{\prime}\right)$ and $\sigma(y)=b=\sigma^{\prime}\left(y^{\prime}\right)$. Let $\gamma$ be the straight line in $I^{m}$ from $x$ to $y$, and let $\gamma^{\prime}$ be the straight line in $I^{n}$ from $x^{\prime}$ to $y^{\prime}$. By Lemma C.2 each of $\sigma \gamma$ and $\sigma^{\prime} \gamma^{\prime}$ is a local geodesic in $C$, and so by [8, II.1.4 (2)] each is a geodesic in $C$. These geodesics have the same endpoints, and so they must coincide. It follows that the intersection of the images of $\sigma$ and $\sigma^{\prime}$ is a convex subset of $C$, and so must be a single face of $\sigma$ and of $\sigma^{\prime}$.

Lemma C.5. Let $C$ be a cube viewed as a cubical complex, and let $\mu$ be a cube of the cubical subdivision $C^{\prime}$ of $C$. The intersection of those faces of $C$ that meet $\mu$ is non-empty.

Proof. There is a natural bijective correspondence between vertices of $C^{\prime}$ and faces of $C$. Containment of faces of $C$ induces a partial ordering on vertices of $C^{\prime}$ such that the vertex set of any cube $\mu$ of $C^{\prime}$ has unique maximal and minimal elements. This establishes a bijection between cubes of $C^{\prime}$ and ordered pairs $\left(\tau_{1}, \tau_{2}\right)$ of faces of $C$ with $\tau_{1} \geq \tau_{2}$ : the pair $\left(\tau_{1}, \tau_{2}\right)$ corresponds to the cube of $\operatorname{dimension} \operatorname{dim}\left(\tau_{1}\right)-\operatorname{dim}\left(\tau_{2}\right)$ whose maximal and minimal vertices are the barycentres of $\tau_{1}$ and $\tau_{2}$. With this notation, $\mu=\left(\tau_{1}, \tau_{2}\right)$ meets $\tau$ if and only if $\tau \geq \tau_{2}$, and in this case the intersection $\mu \cap \tau$ is equal to the cube $\left(\tau_{1} \cap \tau, \tau_{2}\right)$ of $C^{\prime}$. Hence every face of $C$ that meets $\mu=\left(\tau_{1}, \tau_{2}\right)$ contains $\tau_{2}$, and the intersection of all such faces is equal to $\tau_{2}$.

Proposition C.6. Let $C$ be a cube complex with the property that every vertex link in $C$ is a simplicial complex, and let $C^{\prime}, C^{\prime \prime}$ denote the first and second cubical subdivisions of C. Then

(i) Cubes of $C^{\prime}$ embed

(ii) The intersection of any two cubes of $C^{\prime}$ is a disjoint union of cubes of $C^{\prime}$

(iii) $C^{\prime \prime}$ is a cubical complex

REMARK C.7. The link of a vertex of a cube complex is unchanged by passing to the cubical subdivision. So if $C$ has a vertex link that is not simplicial, then so does every cubical subdivision of $C$. 
Proof. Any cube $\mu$ of $C^{\prime}$ is a face of another cube $\nu$ of $C^{\prime}$ such that $\nu$ contains some vertex $v$ of $C$ amongst its vertices. Thus it suffices to show that such a $\nu$ embeds. It suffices to show that the $2^{n}$ vertices of $\nu$ are distinct. The line segments in $\nu$ from $v$ to the other $2^{n}-1$ vertices of $\nu$ define $2^{n}-1$ points of $\operatorname{Lk}_{C^{\prime}}(v)=\operatorname{Lk}_{C}(v)$, and it remains to show that these points are distinct. If $\tau$ is the $(n-1)$-simplex of $\operatorname{Lk}(v)$ defined by $\nu$, then these points are the barycentres of $\tau$ and its faces. Since $\operatorname{Lk}(v)$ is simplicial, these points are distinct. Hence the vertices of $\nu$ lie in the interiors of distinct cubes of $C$, and so they are distinct.

Let $\sigma$ and $\sigma^{\prime}$ be cubes of $C^{\prime}$, and let $v$ be a vertex of the intersection $\sigma \cap \sigma^{\prime}$. Define a set $S$ of vertices of $C^{\prime}$ by

$$
S=\left\{w \in\left(C^{\prime}\right)^{0}:(v, w) \text { is an edge of } \sigma \cap \sigma^{\prime}\right\} .
$$

Since $v$ was an arbitrary vertex of $\sigma \cap \sigma^{\prime}$, it suffices to show that there is a cube contained in $\sigma \cap \sigma^{\prime}$ whose vertex set contains $S \cup\{v\}$. By hypothesis, there is a cube $\mu \leq \sigma$ such that $S \cup\{v\}$ is contained in the vertex set of $\mu$, and we may take $\mu$ of minimal dimension amongst all such cubes, in which case $\mu$ is an $|S|$-cube. Similarly, there is an $|S|$-cube $\mu^{\prime} \leq \sigma^{\prime}$ with $S \cup\{v\}$ contained in its vertex set. The images of $\mu$ and $\mu^{\prime}$ in $\operatorname{Lk}_{C^{\prime}}(v)$ are simplices with the same vertex set. Since $\operatorname{Lk}_{C^{\prime}}(v)$ is simplicial, it follows that $\mu=\mu^{\prime}$, and so $\mu$ is contained in $\sigma \cap \sigma^{\prime}$ as required.

It remains to show that $C^{\prime \prime}$ is cubical. Let $\mu$ be a cube of $C^{\prime \prime}$, and let $\nu$ be a cube of $C^{\prime}$ that contains $\mu$. Since $\nu$ embeds, it follows that $\mu$ embeds. Now let $\mu^{\prime}$ be another cube of $C^{\prime \prime}$, and let $\nu^{\prime}$ be a cube of $C^{\prime}$ that contains $\mu^{\prime}$. The intersection $\mu \cap \mu^{\prime}$ is contained in $\nu \cap \nu^{\prime}$. By Part 2, the intersection $\nu \cap \nu^{\prime}$ is equal to a disjoint union $\sigma_{1} \amalg \cdots \coprod \sigma_{r}$ of cubes of $C^{\prime}$. By Lemma C.5. the set of faces of $\nu$ that meet $\mu$ is a connected subcomplex of $\nu$. It follows that the $\mu$ intersects at most one of the cubes $\sigma_{i}$ non-trivially. Similarly, $\mu^{\prime}$ intersects $\sigma_{j}$ for at most one value of $j$. Hence either $\mu \cap \mu^{\prime}$ is empty, or there exists $i$ so that $\mu \cap \mu^{\prime} \subseteq \sigma_{i}$. In this latter case, since $\sigma_{i}$ embeds in $C^{\prime}$, it follows that $\mu \cap \mu^{\prime}$ is a face of both $\mu$ and $\mu^{\prime}$.

For a vertex $v$ in a cube complex $C$, define $\operatorname{St}_{C}(v)$ to the the union of all of the closed cubes of $C$ that contain $v$. For $m$ a positive integer let $C^{(m)}$ denote the $m$ th iterated cubical subdivision of $C$.

Proposition C.8. For any cube complex $C$ and vertex $v, \operatorname{St}_{C^{\prime \prime}}(v)$ is contractible. For any $m \geq 2$ there is an isomorphism $\phi_{m}: \operatorname{St}_{C^{\prime \prime}}(v) \rightarrow \mathrm{St}_{C^{(m)}}(v)$ which multiplies distances by $2^{2-m}$.

Proof. Let $\sigma$ be an $n$-cube of side length one, and let $V$ be the vertex set of $\sigma$. For each $v \in V$, and each $m \geq 0, \operatorname{St}_{\sigma^{(m)}}(v)$ is an $n$-subcube of $\sigma$ of side length $2^{-m}$ containing $v$ as a vertex. Moreover if $m \geq 2$, then the union

$$
X(\sigma, m)=\bigcup_{v \in V} \operatorname{St}_{\sigma^{(m)}}(v)
$$

is a disjoint union of $2^{n} n$-cubes, each containing a unique vertex from $V$. It follows that there is a linear homotopy $H^{\sigma}$ from the identity map of $X(\sigma, 2)$ to the locally constant map which sends each component of $X(\sigma, 2)$ to the element of $V$ that it contains. If $\tau$ is a face of $\sigma$, then $X(\tau, 2)$ defined similarly is naturally a subspace of $X(\sigma, 2)$ and $H^{\sigma}$ and $H^{\tau}$ agree on $X(\tau, 2)$.

Now if $C$ is any cube complex, define

$$
X(C, m)=\bigcup_{\sigma} X(\sigma, m),
$$

where $\sigma$ ranges over all of the cubes of $C$. By the above remark, the homotopies $H^{\sigma}$ fit together to make a single homotopy $H^{C}$ from the identity map of $X(C, 2)$ to the constant map that sends each component of $X(C, 2)$ to the unique vertex of $C$ that it contains. Since $H^{C}$ was 
defined linearly, if we put $t=1-4 / 2^{m}$, then for any $m \geq 2$, the map $H_{t}^{C}$ is an isomorphism from $X(C, 2)$ to $X(C, m)$, and for any vertex $v$ of $C$ we may define $\phi_{m}$ to be the restriction of $H_{t}^{C}$ to the component $\operatorname{St}_{C^{\prime \prime}}(v)$ of $X(C, 2)$.

Theorem C.9. Let $C$ be a cube complex, with arbitrary vertex links. Then $C$ is locally $C A T(0)$ if and only if all vertex links in $C$ are flag simplicial complexes.

Proof. If $C$ is a cube complex in which all vertex links are flag simplicial complexes, then $C^{\prime \prime}$ is a cubical complex with this property, and the universal cover $\widetilde{C}^{\prime \prime}$ of $C^{\prime \prime}$ satisfies the hypotheses of Theorem B.8. The universal cover $\widetilde{C}$ of $C$ is isometric to $\widetilde{C}^{\prime \prime}$, and so it too is $\mathrm{CAT}(0)$. It follows that $C$ is locally $\mathrm{CAT}(0)$.

Conversely, suppose that $C$ is a cube complex with a vertex $v$ such that the link of $v$ is not a flag simplicial complex. It suffices to show that for all $\epsilon$, the metric ball $B(v, \epsilon)$ around $v$ is not CAT(0). Let $S$ denote $\operatorname{St}_{C^{\prime \prime}}(v)$, and note that $B(v, 1 / 4)$ is contained in $S$. By Proposition C.8 $S$ is contractible, and either $S$ is cubical but has a non-flag link or $S$ is not cubical. It follows from either Theorem C.4 or Theorem B.8 that $S$ cannot be CAT(0). Hence there exists a geodesic triangle $T$ in $S$ that violates the CAT(0) inequality. Now pick any $\epsilon \leq 1 / 12$, and let $m$ be sufficiently large that $\phi_{m}(T)$ is contained in $B(v, \epsilon)$ where $\phi_{m}$ is the map in Proposition C.8. Since $\phi_{m}(T)$ violates the CAT(0) inequality in $\operatorname{St}_{C^{(m)}}(v)$, it suffices to show that the embedding of $\phi_{m}(T)$ into $C$ is isometric. Since $\phi_{m}(T)$ is contained in $B(v, \epsilon)$, it follows that the distance in $\mathrm{St}_{C^{(m)}}$ between any two points of $\phi_{m}(T)$ is at most $2 \epsilon \leq 1 / 6$. If there were a shorter path in $C$ between two points of $\phi_{m}(T)$, it could not leave $B(v, 1 / 4)$. Since $B(v, 1 / 4) \subset \operatorname{St}_{C^{\prime \prime}}(v)$ and the inclusion of $\operatorname{St}_{C^{(m)}}(v)$ into $\operatorname{St}_{C^{\prime \prime}}(v)$ is an isometry, it follows that $\phi_{m}(T)$ embeds isometrically into $C$, and so $B(v, \epsilon)$ is not $\operatorname{CAT}(0)$.

REmark C.10. The special case of Theorem C.9 in which $C$ is assumed to be finitedimensional is given in Gromov's original paper [11, section 4.2.C] and in [8, theorem II.5.20].

Corollary C.11. The second cubical subdivision of any locally CAT(0) cube complex is cubical.

Proof. If $C$ is a locally CAT(0) cube complex, then Theorem C.9 shows that $C$ satisfies the hypotheses of Proposition C.6. and hence $C^{\prime \prime}$ is cubical.

\section{References}

1. A. Bartels, S. Echterhoff, and W. Lück. Inheritance of isomorphism conjectures under colimits. In $K$-theory and noncommutative geometry, EMS Ser. Congr. Rep., pages 41-70. Eur. Math. Soc., Zürich, 2008.

2. A. Bartels and W. Lück. The Borel conjecture for hyperbolic and CAT(0)-groups, 2009.

3. G. Baumslag, E. Dyer, and A. Heller. The topology of discrete groups. J. Pure Appl. Algebra, 16(1):1-47, 1980.

4. M. Bestvina. Questions in geometric group theory, 2004.

5. J. Block. Some remarks concerning the Baum-Connes conjecture. Comm. Pure Appl. Math., 50(9):813-820, 1997.

6. N. Brady, I. J. Leary, and B. E. A. Nucinkis. On algebraic and geometric dimensions for groups with torsion. J. London Math. Soc. (2), 64(2):489-500, 2001.

7. M. R. Bridson. Geodesics and curvature in metric simplicial complexes. In Group theory from a geometrical viewpoint (Trieste, 1990), pages 373-463. World Sci. Publ., River Edge, NJ, 1991.

8. M. R. Bridson and A. Haefliger. Metric spaces of non-positive curvature, volume 319 of Grundlehren der Mathematischen Wissenschaften [Fundamental Principles of Mathematical Sciences]. Springer-Verlag, Berlin, 1999. 
9. R. M. Charney and M. W. Davis. Strict hyperbolization. Topology, 34(2):329-350, 1995.

10. C. H. Dowker. Topology of metric complexes. Amer. J. Math., 74:555-577, 1952.

11. M. Gromov. Hyperbolic groups. In Essays in group theory, volume 8 of Math. Sci. Res. Inst. Publ., pages 75-263. Springer, New York, 1987.

12. A. Hatcher. Algebraic topology. Cambridge University Press, Cambridge, 2002.

13. J.-C. Hausmann. Every finite complex has the homology of a duality group. Math. Ann., 275(2):327-336, 1986.

14. N. Higson and G. Kasparov. Operator $K$-theory for groups which act properly and isometrically on Hilbert space. Electron. Res. Announc. Amer. Math. Soc., 3:131-142 (electronic), 1997.

15. T. Januszkiewicz and J. Świątkowski. Simplicial nonpositive curvature. Publ. Math. Inst. Hautes Études Sci., 104:1-85, 2006.

16. D. M. Kan and W. P. Thurston. Every connected space has the homology of a $K(\pi, 1)$. Topology, 15(3):253-258, 1976.

17. R. Kim. Every finite complex has the homology of some cat(0) cubical duality group, 2012.

18. V. Lafforgue. $K$-théorie bivariante pour les algèbres de Banach et conjecture de Baum-Connes. Invent. Math., 149(1):1-95, 2002.

19. I. J. Leary and B. E. A. Nucinkis. Every CW-complex is a classifying space for proper bundles. Topology, 40(3):539-550, 2001.

20. W. Lück. Chern characters for proper equivariant homology theories and applications to $K$ - and $L$-theory. J. Reine Angew. Math., 543:193-234, 2002.

21. W. Lück. On the Farrell-Jones and related conjectures. In Cohomology of groups and algebraic $K$-theory, volume 12 of Adv. Lect. Math. (ALM), pages 269-341. Int. Press, Somerville, MA, 2010.

22. C. R. F. Maunder. A short proof of a theorem of Kan and Thurston. Bull. London Math. Soc., 13(4):325$327,1981$.

23. D. McDuff. On the classifying spaces of discrete monoids. Topology, 18(4):313-320, 1979.

24. G. Mislin and A. Valette. Proper group actions and the Baum-Connes conjecture. Advanced Courses in Mathematics. CRM Barcelona. Birkhäuser Verlag, Basel, 2003.

25. G. Moussong. Hyperbolic Coxeter Groups. PhD thesis, Ohio State, 1988.

26. G. A. Niblo and L. D. Reeves. Groups acting on CAT(0) cube complexes. Geom. Topol., 1:approx. 7 pp. (electronic), 1997.

27. G. A. Niblo and L. D. Reeves. The geometry of cube complexes and the complexity of their fundamental groups. Topology, 37(3):621-633, 1998.

28. J. K. C. Polák and D. T. Wise. A note on vh subdivisions, 2012.

29. D. Quillen. The spectrum of an equivariant cohomology ring. I, II. Ann. of Math. (2), 94:549-572; ibid. (2) 94 (1971), 573-602, 1971.

30. C. P. Rourke and B. J. Sanderson. $\triangle$-sets. I. Homotopy theory. Quart. J. Math. Oxford Ser. (2), 22:321-338, 1971.

31. M. Sageev. Ends of group pairs and non-positively curved cube complexes. Proc. London Math. Soc. (3), 71(3):585-617, 1995.

32. C. Wegner. The $K$-theoretic Farrell-Jones conjecture for CAT(0)-groups. Proc. Amer. Math. Soc., 140(3):779-793, 2012.

33. G. W. Whitehead. Elements of homotopy theory, volume 61 of Graduate Texts in Mathematics. SpringerVerlag, New York, 1978.

Ian J. Leary

Department of Mathematics

The Ohio State University

231 West 18th Avenue

Columbus

Ohio 43210

USA

Current address:

School of Mathematics

University of Southampton

Southampton

SO17 1BJ

UK

i.j.leary@soton.ac.uk 